\title{
948.
}

\section{REPORT OF A COMMITTEE APPOINTED FOR THE PURPOSE OF CARRYING ON THE TABLES CONNECTED WITH THE PELLIAN EQUATION FROM THE POINT WHERE THE WORK WAS LEFT BY DEGEN IN 1817.}

[From the British Association Report, (1893), pp. 73-120.]

WE have, on the Pellian Equation, Degen's tables, the title of which is "Canon Pellianus sive Tabula simplicissimam æquationis celebratissimæ $y^{2}=a x^{2}+1$ solutionem pro singulis numeri dati valoribus ab 1 usque ad 1000 in numeris rationalibus iisdemque integris exhibens." Autore Carolo Ferdinando Degen. Hafniæ, apud Gerhardum Bonnierum, MDcccxviI., 8vo. Introductio, pp. v-xxiv. Tabula I. Solutionem æquationis $y^{2}-a x^{2}-1=0$ exhibens, pp. 3-106. Tabula II. Solutionem æquationis $y^{2}-a x^{2}+1=0$, quotiescunque valor ipsius $a$ talem admiserit, exhibens, pp. 109-112.

The mode of calculation is explained in the Introduction, and illustrated by the examples of the numbers 209, 173.

As to the first of these, the entry in Table I. is

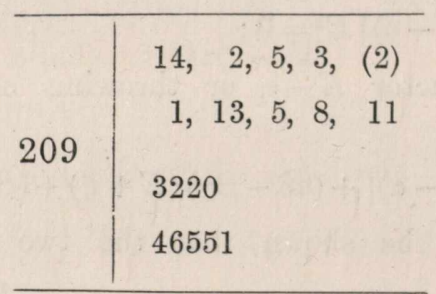

where the first line gives the expression of $\sqrt{209}$ as a continued fraction, viz. we have

$$
\sqrt{209}=14+\frac{1}{2}+\frac{1}{5}+\frac{1}{3}+\frac{1}{2}+\frac{1}{3}+\frac{1}{5}+\frac{1}{2}+\frac{1}{28}+\frac{1}{2}+\& c .
$$


the denominators being $2,5,3,(2), 3,5,2$, then 28 , which is the double of the integer part 14, and then again. 2, 5, 3, (2), 3, 5, 2, and so on, the parentheses of the (2) being used to indicate that this is the middle term of the period.

The second row gives auxiliary numbers occurring in the calculation of the first row and having a meaning, as will presently appear. Observe that the 11 which comes under the (2) should also be printed in parentheses (11); but this is not done.

The process for the calculation of the $x, y$ is as follows:

\begin{tabular}{|r|r|r|r|}
\hline \multicolumn{5}{|c|}{209} & \\
\hline 14 & 1 & 0 & +1 \\
2 & 14 & 1 & -13 \\
5 & 29 & 2 & +5 \\
3 & 159 & 11 & -8 \\
$(2)$ & 506 & 35 & $+(11)$ \\
3 & 1171 & 81 & -8 \\
5 & 4019 & 278 & +5 \\
2 & 21266 & 1471 & -13 \\
28 & 46551 & 3220 & +1 \\
\hline
\end{tabular}

viz. writing down as a first column the numbers of the first row, and beginning the second column with 1,14 (14 the number at the head of the first column), and the third column with 0,1 , we calculate the numbers of the second column, $29=2.14+1$, $159=5.29+14,506=3.159+29$, \&c., and the numbers of the third column in like manner, $2=2.1+0,11=5.2+1,35=3.11+2$, \&c.; and then writing down as a fourth column the numbers of the second row with the signs + , - alternately, we have a series of equations $y^{2}-a x^{2}= \pm A$, viz.

$$
\begin{aligned}
1^{2}-209.0^{2} & =+1, \\
14^{2}-209.1^{2} & =-13, \\
29^{2}-209.2^{2} & =+5,
\end{aligned}
$$

the last of them being

$$
(46551)^{2}-209(3220)^{2}=+1
$$

this last corresponding as above to the value +1 , and the numbers 46551 and 3220 being accordingly the $y$ and $x$ given in the fourth and third rows of the table. 
As to the second of the foregoing numbers, 173, the only difference is that the period has a double middle term, viz. the entry in the Table I. is

\begin{tabular}{l|l}
\hline \multirow{1}{*}{173} & $13,6,(1,1)$ \\
& $1,4,(13,13)$ \\
& 190060 \\
& 2499849 \\
\hline
\end{tabular}

The first row gives the expression of $\sqrt{173}$, viz. that is

$$
\sqrt{173}=13+\frac{1}{6}+\frac{1}{(1)}+\frac{1}{(1)}+\frac{1}{6}+\frac{1}{26}+\& c .
$$

the denominators being $6,1,1,6$, then 26 (the double of the integer part 13), and then again $6,1,1,6$, and so on. In the second row I remark that Degen prints the parentheses $(13,13)$ for the double middle term.

The process for the calculation of the $x, y$ is similar to that in the former case, viz. we have

\begin{tabular}{|r|r|r|r|}
\hline \multicolumn{4}{|c|}{173} \\
\hline 13 & 1 & 0 & +1 \\
6 & 13 & 1 & -4 \\
$\left(\begin{array}{l}1 \\
1\end{array}\right)$ & 79 & 6 & +13 \\
6 & 92 & 7 & -13 \\
26 & 171 & 13 & +4 \\
\hline
\end{tabular}

where the second and third columns begin 1,13 and 0,1 respectively, and the remaining terms are calculated $79=6.13+1,92=1.79+13$, \&c., and $6=6.1+0$, $7=1.6+1$, \&c.; and then writing down as a fourth column the terms of the second row with the signs + , - alternately, we have

$$
\begin{array}{r}
1^{2}-173.0^{2}=+1 \\
13^{2}-173.1^{2}=-4 \\
79^{2}-173.6^{2}=+13
\end{array}
$$

the last equation being

$$
(1118)^{2}-173(85)^{2}=-1 \text {, }
$$


the term for the last equation being always in a case such as the present one, not +1 , but -1 . The final numbers 1118,85 are consequently entered not in Table I., but in Table II., viz. the entry in this table is

\begin{tabular}{l|l}
\hline 173 & 85 \\
& 1118 \\
\hline
\end{tabular}

and thence we calculate the numbers $y, x$ of Table I., viz, these are

$$
\begin{aligned}
2499849 & =2 .(1118)^{2}+1 \\
190060 & =2.1118 .85 .
\end{aligned}
$$

Generally Table II. gives for each value of $a$, comprised therein, values of $x, y$, such that $y^{2}=a x^{2}-1$, and then writing $y_{1}=2 y^{2}+1, x_{1}=2 x y$, we have

$$
y_{1}^{2}=\left(2 a x^{2}-1\right)^{2}=4 a^{2} x^{4}-4 a x^{2}+1=a \cdot 4 x^{2}\left(a x^{2}-1,+1=a x_{1}^{2}+1,\right.
$$

so that $x_{1}, y_{1}$ are for the same value of $a$ the values of $x, y$ in Table I.

It is to be remarked that the heading of Table II. is not perfectly accurate, for it purports to give for every value of $a$, for which a solution exists, a solution of the equation $y^{2}=a x^{2}-1$. What it really gives is the solution for each value of $a$ for which the period has a double middle term. But if $a=\alpha^{2}+1$, then obviously we have a solution $y=\alpha, x=1$, and for any such value of $a$ the period has a single middle term, viz. the entry in Table I. is

\begin{tabular}{l|l}
\hline$a^{2}+1$ & $\alpha,(2 \alpha)$ \\
& $1, \quad 1$ \\
$2 \alpha$ \\
$2 \alpha^{2}+1$ \\
\hline
\end{tabular}

and we, in fact, have

\begin{tabular}{|c|r|r|r|}
\hline \multicolumn{4}{|c|}{$a^{2}+1$} \\
\hline$a$ & 1 & 0 & +1 \\
$(2 \alpha)$ & $a$ & 1 & -1 \\
$2 a$ & $2 \alpha^{2}+1$ & $2 a$ & +1 \\
\hline
\end{tabular}

that is,

$$
\begin{array}{r}
1^{2}-\left(\alpha^{2}+1\right) 0^{2}=+1 \\
\alpha^{2}-\left(\alpha^{2}+1\right) 1^{2}=-1 \\
\left(2 \alpha^{2}+1\right)^{2}-\left(\alpha^{2}+1\right)(2 \alpha)^{2}=+1
\end{array}
$$

C. XIII. 
The foregoing instances of the calculation of $x, y$ in the case of the numbers 209 and 173 suggest a table which may be regarded as an extended form of Degen's tables; viz. such a table, from $a=2$ to $a=99$, is as follows:

Specimen of extended Form of Table in regard to the Peldian Equation.

\begin{tabular}{|c|c|c|c|c|c|c|c|c|c|}
\hline$a$ & & $y$ & $x$ & $y^{2}-a x^{2}$ & $a$ & & $y$ & $x$ & $y^{2}-a x^{2}$ \\
\hline \multirow[t]{3}{*}{2} & 1 & 1 & 0 & +1 & \multirow[t]{6}{*}{13} & 3 & 1 & 0 & +1 \\
\hline & (2) & 1 & 1 & -1 & & 1 & 3 & 1 & -4 \\
\hline & & 3 & 2 & +1 & & $(1)$ & 4 & 1 & +3 \\
\hline \multirow[t]{3}{*}{3} & 1 & 1 & 0 & +1 & & (1) & 7 & $\begin{array}{l}2 \\
3\end{array}$ & \\
\hline & (1) & 1 & 1 & -2 & & 6 & 18 & 5 & -1 \\
\hline & 2 & 2 & 1 & +1 & & & & & \\
\hline \multirow[t]{3}{*}{5} & 2 & 1 & 0 & +1 & \multirow[t]{5}{*}{14} & 3 & 1 & 0 & +1 \\
\hline & (4) & 2 & 1 & -1 & & 1 & 3 & 1 & -5 \\
\hline & 4 & 9 & 4 & +1 & & (2) & 4 & 1 & +2 \\
\hline \multirow{4}{*}{6} & & & & & & & 11 & & -0 \\
\hline & 2 & 1 & 0 & +1 & & 6 & 15 & 4 & +1 \\
\hline & (2) & 2 & 1 & -2 & \multirow{3}{*}{15} & 3 & 1 & 0 & +1 \\
\hline & 4 & 5 & 2 & +1 & & (1) & 3 & 1 & -6 \\
\hline \multirow[t]{5}{*}{7} & 2 & 1 & 0 & +1 & & 6 & 4 & 1 & +1 \\
\hline & 1 & 2 & 1 & -3 & \multirow{4}{*}{17} & & & 0 & \\
\hline & (1) & 3 & 1 & +2 & & 4 & 1 & 1 & -1 \\
\hline & 1 & 5 & 2 & -3 & & (8) & 4 & & \\
\hline & 4 & 8 & 3 & +1 & & & 33 & & \\
\hline \multirow[t]{3}{*}{8} & 2 & 1 & 0 & +1 & \multirow[t]{3}{*}{18} & 4 & 1 & 0 & +1 \\
\hline & (1) & 2 & 1 & -4 & & (4) & 4 & - & -2 \\
\hline & 4 & 3 & 1 & +1 & & & 17 & 4 & +1 \\
\hline \multirow[t]{3}{*}{10} & 3 & 1 & 0 & +1 & \multirow[t]{6}{*}{19} & 4 & 1 & 0 & \\
\hline & (6) & 3 & 1 & -1 & & 2 & 4 & 1 & \\
\hline & 6 & 19 & 6 & +1 & & 1 & 9 & 2 & +5 \\
\hline \multirow{3}{*}{11} & & & & 7 & & (ग) & 13 & 11 & $\begin{array}{r}-2 \\
+5\end{array}$ \\
\hline & (3) & 3 & 1 & $\begin{array}{l}-\quad 2 \\
-\end{array}$ & & 2 & 61 & 14 & -3 \\
\hline & 6 & 10 & 3 & +1 & & 8 & 170 & 39 & +1 \\
\hline \multirow[t]{3}{*}{12} & 3 & 1 & 0 & +1 & \multirow[t]{3}{*}{20} & 4 & 1 & 0 & +1 \\
\hline & (2) & 3 & 1 & -3 & & (2) & 4 & 1 & -4 \\
\hline & 6 & 7 & 2 & +1 & & 8 & 9 & 2 & +1 \\
\hline
\end{tabular}


Specimen of extended Form of Pellian Equation Table-continued.

\begin{tabular}{|c|c|c|c|c|c|c|c|c|c|}
\hline$a$ & & $y$ & $x$ & $y^{2}-a x$ & $a$ & & $y$ & $x$ & $y^{2}-a x^{2}$ \\
\hline \multirow[t]{7}{*}{21} & 4 & 1 & 0 & +1 & \multirow[t]{6}{*}{29} & 5 & 1 & 0 & +1 \\
\hline & 1 & 4 & 1 & -5 & & 2 & 5 & 1 & -6 \\
\hline & 1 & 5 & 1 & +4 & & $(1)$ & 11 & 2 & +5 \\
\hline & (2) & 9 & 2 & -3 & & (1) & 16 & 3 & -3 \\
\hline & 1 & 23 & 5 & +4 & & 2 & 27 & 5 & +2 \\
\hline & 1 & 32 & 7 & -5 & & 10 & 70 & 13 & -1 \\
\hline & 8 & 55 & 12 & +1 & \multirow{4}{*}{30} & & & & \\
\hline \multirow[t]{7}{*}{22} & 4 & 1 & 0 & +1 & & (2) & 5 & 1 & -5 \\
\hline & 1 & 4 & 1 & -6 & & 10 & 11 & 2 & +1 \\
\hline & 2 & 5 & 1 & +3 & & & & & \\
\hline & (4) & 14 & 3 & -2 & \multirow[t]{9}{*}{31} & 5 & 1 & 0 & +1 \\
\hline & 2 & 61 & 13 & +3 & & 1 & 5 & 1 & -6 \\
\hline & 1 & 136 & 29 & -6 & & 1 & 6 & 1 & +5 \\
\hline & 8 & 197 & 42 & +1 & & 3 & 11 & 2 & -3 \\
\hline \multirow[t]{5}{*}{23} & 4 & 1 & 0 & +1 & & $\begin{array}{c}(5) \\
3\end{array}$ & $\begin{array}{r}39 \\
206\end{array}$ & $\begin{array}{r}7 \\
37\end{array}$ & $\begin{array}{r}+2 \\
-\quad 3\end{array}$ \\
\hline & 1 & 4 & 1 & -7 & & 1 & 657 & $\begin{array}{r}318 \\
118\end{array}$ & $\begin{array}{r}-3 \\
+\quad 5\end{array}$ \\
\hline & (3) & 5 & 1 & +2 & & 1 & 863 & 155 & \\
\hline & 1 & 19 & 4 & -7 & & 10 & 1520 & 273 & $\begin{array}{r}-6 \\
+\quad 1\end{array}$ \\
\hline & 8 & 24 & 5 & +1 & & & & & \\
\hline \multirow[t]{3}{*}{24} & 4 & 1 & 0 & +1 & \multirow[t]{5}{*}{32} & 5 & 1 & 0 & \\
\hline & (1) & 4 & 1 & -8 & & 1 & 5 & 1 & \\
\hline & 8 & 5 & 1 & +1 & & (1) & 6 & 1 & +4 \\
\hline \multirow{3}{*}{26} & & & & & & 1 & 11 & 2 & -7 \\
\hline & 5 & 1 & 0 & & & & & 3 & +1 \\
\hline & 10 & 51 & 10 & $\begin{array}{r}+1 \\
+\quad 1\end{array}$ & \multirow[t]{4}{*}{33} & 5 & 1 & 0 & \\
\hline \multirow{3}{*}{27} & 5 & 1 & 0 & +1 & & $\begin{array}{c}1 \\
(2)\end{array}$ & $\begin{array}{l}5 \\
6\end{array}$ & $\begin{array}{l}1 \\
1\end{array}$ & $\begin{array}{l}-8 \\
+\quad 3\end{array}$ \\
\hline & (5) & 5 & 1 & -2 & & 1 & 17 & 3 & -8 \\
\hline & 10 & 26 & 5 & +1 & & 10 & 23 & 4 & +1 \\
\hline \multirow[t]{5}{*}{28} & 5 & 1 & 0 & +1 & \multirow[t]{5}{*}{34} & 5 & 1 & 0 & +1 \\
\hline & 3 & 5 & 1 & -3 & & 1 & 5 & 1 & -9 \\
\hline & (2) & 16 & 3 & +4 & & (4) & 6 & 1 & +2 \\
\hline & 3 & 37 & 7 & -3 & & 1 & 29 & 5 & -9 \\
\hline & 10 & 127 & 24 & +1 & & 10 & 35 & 6 & +1 \\
\hline
\end{tabular}


Specimen of extended Form of Pellian Equation Table-continued.

\begin{tabular}{|c|c|c|c|c|c|c|c|c|c|}
\hline$a$ & & $y$ & $x$ & $y^{2}-a x^{2}$ & $a$ & & $y$ & $x$ & $y^{2}-a x^{2}$ \\
\hline \multirow[t]{3}{*}{35} & 5 & 1 & 0 & +1 & \multirow[t]{8}{*}{44} & 6 & 1 & 0 & +1 \\
\hline & (1) & 5 & 1 & -10 & & 1 & 6 & 1 & -8 \\
\hline & 10 & 6 & 1 & +1 & & 1 & 7 & 1 & +5 \\
\hline \multirow[t]{3}{*}{37} & 6 & 1 & 0 & +1 & & $\begin{array}{c}1 \\
(2)\end{array}$ & $\begin{array}{l}13 \\
20\end{array}$ & $\begin{array}{l}2 \\
3\end{array}$ & $\begin{array}{r}-7 \\
+\quad 4\end{array}$ \\
\hline & (12) & 6 & 1 & -1 & & $\begin{array}{l}\text { (2) } \\
1\end{array}$ & 53 & 8 & $\begin{array}{r}1 \\
-\quad 7\end{array}$ \\
\hline & 12 & 73 & 12 & +1 & & 1 & 73 & 11 & +5 \\
\hline \multirow{4}{*}{38} & 6 & 1 & 0 & +1 & & 1 & 126 & 19 & -8 \\
\hline & (6) & 6 & 1 & -2 & & 12 & 199 & 30 & +1 \\
\hline & 12 & 37 & 6 & +1 & \multirow[t]{6}{*}{45} & 6 & 1 & 0 & +1 \\
\hline & & & & & & 1 & 6 & 1 & -9 \\
\hline \multirow[t]{3}{*}{39} & 6 & 1 & 0 & +1 & & 2 & 7 & 1 & +4 \\
\hline & (4) & 6 & 1 & -3 & & (2) & 20 & 3 & -5 \\
\hline & 12 & 25 & 4 & +1 & & 2 & 47 & 7 & +4 \\
\hline \multirow[t]{3}{*}{40} & 6 & 1 & 0 & +1 & & $\begin{array}{r}1 \\
12\end{array}$ & $\begin{array}{l}114 \\
161\end{array}$ & $\begin{array}{l}17 \\
24\end{array}$ & $\begin{array}{l}-9 \\
+\quad 1\end{array}$ \\
\hline & (3) & 6 & 1 & -4 & \multirow[t]{12}{*}{46} & 6 & 1 & 0 & +1 \\
\hline & 12 & 19 & 3 & +1 & & 1 & 6 & 1 & -10 \\
\hline \multirow[t]{4}{*}{41} & 6 & 1 & 0 & +1 & & 3 & 7 & 1 & +3 \\
\hline & $(2)$ & 6 & 1 & -5 & & 1 & 27 & . 4 & -7 \\
\hline & (2) & 13 & 2 & +5 & & 1 & 34 & 5 & +6 \\
\hline & 12 & 32 & 5 & -1 & & 2 & 61 & 9 & -5 \\
\hline \multirow[t]{3}{*}{42} & 6 & 1 & 0 & +1 & & $\begin{array}{c}(6) \\
2\end{array}$ & $\begin{array}{l}156 \\
997\end{array}$ & $\begin{array}{r}23 \\
147\end{array}$ & $\begin{array}{r}+\quad 2 \\
-\quad 5\end{array}$ \\
\hline & (2) & 6 & 1 & -6 & & 1 & 2150 & 317 & $\begin{array}{r}0 \\
+\quad 6\end{array}$ \\
\hline & 12 & 13 & 2 & +1 & & 1 & 3147 & 464 & -7 \\
\hline \multirow{11}{*}{43} & 6 & 1 & 0 & +1 & & 3 & 5297 & 781 & +3 \\
\hline & 1 & 6 & 1 & $\begin{array}{r}1 \\
-\quad 7\end{array}$ & & 1 & 19038 & 2807 & -10 \\
\hline & 1 & 7 & 1 & +6 & & 12 & 24335 & 3588 & +1 \\
\hline & 3 & 13 & 2 & -3 & \multirow[t]{5}{*}{47} & 6 & 1 & 0 & +1 \\
\hline & 1 & 46 & 7 & +9 & & 1 & 6 & 1 & -1 \\
\hline & (5) & 59 & 9 & -2 & & (5) & 7 & 1 & +2 \\
\hline & 1 & 341 & 52 & +9 & & 1 & 41 & 6 & -11 \\
\hline & 3 & 400 & 61 & -3 & & 12 & 48 & 7 & +1 \\
\hline & 1 & 1541 & 235 & +6 & \multirow[t]{3}{*}{48} & 6 & 1 & 0 & +1 \\
\hline & 1 & 1941 & 296 & -7 & & (1) & 6 & 1 & -12 \\
\hline & 12 & 3482 & 531 & +1 & & 12 & 7 & 1 & +1 \\
\hline
\end{tabular}


Specimen of extended Form of Pellian Equation Table-continued.

\begin{tabular}{|c|c|c|c|c|c|c|c|c|c|}
\hline$a$ & & $y$ & $x$ & $y^{2}-a x^{2}$ & $a$ & & $y$ & $x$ & $y^{2}-a x^{2}$ \\
\hline \multirow[t]{3}{*}{50} & 7 & 1 & 0 & +1 & \multirow[t]{6}{*}{57} & 7 & 1 & 0 & +1 \\
\hline & (14) & 7 & 1 & -1 & & 1 & 7 & 1 & -8 \\
\hline & 14 & 99 & 14 & +1 & & 1 & 8 & 1 & +7 \\
\hline \multirow[t]{3}{*}{51} & 7 & 1 & 0 & +1 & & $\begin{array}{c}\text { (4) } \\
1\end{array}$ & $\begin{array}{l}15 \\
68\end{array}$ & $\begin{array}{l}2 \\
9\end{array}$ & -3 \\
\hline & (7) & 7 & 1 & -2 & & 1 & $\begin{array}{l}08 \\
83\end{array}$ & 11 & $\begin{array}{l}+1 \\
-8\end{array}$ \\
\hline & 14 & 50 & 7 & +1 & & 14 & 151 & 20 & $\begin{array}{r}1 \\
+\quad 1\end{array}$ \\
\hline \multirow[t]{7}{*}{52} & 7 & 1 & 0 & +1 & \multirow{8}{*}{58} & 7 & 1 & 0 & \\
\hline & 4 & 7 & 1 & -3 & & 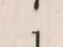 & 7 & 1 & $\begin{array}{l}+1 \\
-\quad 9\end{array}$ \\
\hline & 1 & 29 & 4 & +9 & & 1 & 8 & 1 & $\begin{array}{r}0 \\
+\quad 6\end{array}$ \\
\hline & (2) & 36 & 5 & -4 & & (1) & 15 & 2 & -7 \\
\hline & 1 & 101 & 14 & +9 & & $(1)$ & 23 & 3 & +7 \\
\hline & 4 & 137 & 19 & -3 & & 1 & 38 & 5 & -6 \\
\hline & 14 & 649 & 90 & +1 & & 1 & 61 & 8 & +9 \\
\hline \multirow[t]{6}{*}{53} & 7 & 1 & 0 & +1 & & 14 & 99 & 13 & -1 \\
\hline & 3 & 7 & 1 & -4 & \multirow{8}{*}{59} & & & & +1 \\
\hline & $\left(\begin{array}{l}1 \\
)\end{array}\right.$ & 22 & 3 & +7 & & 7 & i & 1 & -10 \\
\hline & (1) & 29 & 4 & -7 & & $\begin{array}{l}1 \\
2\end{array}$ & 8 & $\begin{array}{l}1 \\
1\end{array}$ & $\begin{array}{r}0 \\
+\quad 5\end{array}$ \\
\hline & 14 & $\begin{array}{r}51 \\
182\end{array}$ & $\begin{array}{r}7 \\
85\end{array}$ & +4 & & (7) & 23 & 3 & -2 \\
\hline & & & 20 & & & 2 & 169 & 22 & +5 \\
\hline \multirow[t]{7}{*}{54} & 7 & 1 & 0 & +1 & & 1 & 361 & 47 & -10 \\
\hline & 2 & 7 & 1 & -5 & & 14 & 530 & 69 & +1 \\
\hline & 1 & 15 & 2 & +9 & & & & & \\
\hline & (6) & 22 & 3 & -2 & \multirow[t]{5}{*}{60} & 7 & 1 & 0 & +1 \\
\hline & 1 & 147 & 20 & +9 & & 1 & 7 & 1 & -11 \\
\hline & 2 & 169 & 23 & -5 & & (2) & 8 & 1 & +4 \\
\hline & 14 & 485 & 66 & +1 & & 1 & 23 & 3 & -11 \\
\hline \multirow{5}{*}{55} & 7 & 1 & 0 & +1 & & 14 & 31 & 4 & +1 \\
\hline & 2 & 7 & 1 & -6 & \multirow[t]{8}{*}{61} & 7 & 1 & 0 & +1 \\
\hline & (2) & 15 & 2 & +5 & & 1 & 7 & 1 & -12 \\
\hline & 2 & 37 & 5 & -6 & & 4 & 8 & 1 & +3 \\
\hline & 14 & 89 & 12 & +1 & & 3 & 39 & 5 & -4 \\
\hline \multirow{4}{*}{56} & & & & & & 1 & 125 & 16 & +9 \\
\hline & 7 & 1 & 0 & +1 & & $\left({ }^{2}\right)$ & 164 & 21 & -5 \\
\hline & (2) & 7 & 1 & -7 & & (2) & 453 & 58 & +5 \\
\hline & 14 & 15 & 2 & +1 & & 1 & 1070 & 137 & -9 \\
\hline
\end{tabular}


Specimen of extended Form of Pellian Equation Table-continued.

\begin{tabular}{|c|c|c|c|c|c|c|c|c|c|}
\hline$a$ & & $y$ & $x$ & $y^{2}-a x^{2}$ & $a$ & & $y$ & $x$ & $y^{2}-a x^{2}$ \\
\hline & 3 & 1523 & 195 & +4 & & 3 & 25 & 3 & +4 \\
\hline & 4 & 5639 & 722 & -3 & & 1 & 83 & 10 & -11 \\
\hline & 1 & 24079 & 3083 & +12 & & (4) & 108 & 13 & +3 \\
\hline & 14 & 29718 & 3805 & -1 & & 1 & 515 & 62 & -11 \\
\hline \multirow[t]{5}{*}{62} & 7 & 1 & 0 & +1 & & 3 & 623 & $\begin{array}{r}75 \\
907\end{array}$ & +4 \\
\hline & 1 & 7 & 1 & -13 & & $\begin{array}{r}3 \\
3\end{array}$ & 2384 & 297 & -5 \\
\hline & (6) & 8 & 1 & +2 & & 16 & 7775 & 936 & +1 \\
\hline & 1 & 55 & 7 & -13 & \multirow[t]{6}{*}{70} & 8 & 1 & 0 & +1 \\
\hline & 14 & 63 & 8 & +1 & & 2 & 8 & 1 & -6 \\
\hline \multirow[t]{3}{*}{63} & 7 & 1 & 0 & +1 & & $\begin{array}{c}1 \\
(2)\end{array}$ & $\begin{array}{l}17 \\
25\end{array}$ & $\begin{array}{l}2 \\
3\end{array}$ & $\begin{array}{l}+9 \\
-\quad 5\end{array}$ \\
\hline & (1) & 7 & 1 & -14 & & 1 & 67 & 8 & $\begin{array}{r}+9 \\
+\quad 9\end{array}$ \\
\hline & 14 & 8 & 1 & +1 & & 2 & 92 & 11 & -6 \\
\hline \multirow[t]{3}{*}{65} & 8 & 1 & 0 & +1 & & 16 & 251 & 30 & +1 \\
\hline & (16) & 8 & 1 & -1 & \multirow[t]{8}{*}{71} & 8 & 1 & 0 & +1 \\
\hline & 16 & 129 & 16 & +1 & & 2 & 8 & 1 & -7 \\
\hline \multirow[t]{3}{*}{66} & 8 & 1 & 0 & +1 & & $\begin{array}{l}2 \\
1\end{array}$ & $\begin{array}{l}17 \\
42\end{array}$ & 2 & +5 \\
\hline & (8) & 8 & 1 & -2 & & (7) & 59 & $\begin{array}{l}5 \\
7\end{array}$ & $\begin{array}{r}-11 \\
+\quad 2\end{array}$ \\
\hline & 16 & 65 & 8 & +1 & & 1 & 455 & 54 & -11 \\
\hline \multirow[t]{11}{*}{67} & 8 & 1 & 0 & +1 & & 2 & 514 & 61 & +5 \\
\hline & 5 & 8 & 1 & $\begin{array}{r}-3 \\
-\quad 3\end{array}$ & & 2 & 1483 & 176 & -7 \\
\hline & 2 & 41 & 5 & $\begin{array}{r}6 \\
+\quad 6\end{array}$ & & 16 & 3480 & 413 & +1 \\
\hline & 1 & 90 & 11 & -7 & \multirow[t]{3}{*}{72} & 8 & 1 & 0 & +1 \\
\hline & 1 & 131 & 16 & +9 & & (2) & 8 & 1 & -3 \\
\hline & (7) & 221 & 27 & -2 & & 16 & 17 & 2 & +1 \\
\hline & 1 & 1678 & 205 & +9 & & & & & \\
\hline & 1 & 1899 & 232 & -7 & \multirow[t]{7}{*}{73} & 8 & 1 & 0 & +1 \\
\hline & 2 & 3577 & 437 & +6 & & 1 & 8 & 1 & -9 \\
\hline & 5 & 9053 & 1106 & -3 & & 1 & 9 & 1 & +8 \\
\hline & 16 & 48842 & 5967 & +1 & & $\left({ }^{5}\right)$ & 17 & 2 & -3 \\
\hline \multirow[t]{3}{*}{68} & 8 & 1 & 0 & +1 & & $\begin{array}{c}(5) \\
1\end{array}$ & $\begin{array}{r}94 \\
487\end{array}$ & $\begin{array}{l}11 \\
57\end{array}$ & $\begin{array}{l}+3 \\
-\quad 8\end{array}$ \\
\hline & (4) & 8 & 1 & -4 & & 1 & 581 & 68 & $\begin{array}{r}+9 \\
+\quad 9\end{array}$ \\
\hline & 16 & 33 & 4 & +1 & & 16 & 1068 & 125 & -1 \\
\hline \multirow[t]{2}{*}{69} & 8 & 1 & 0 & +1 & \multirow[t]{2}{*}{74} & 8 & 1 & 0 & +1 \\
\hline & 3 & 8 & 1 & -5 & & 1 & 8 & 1 & -10 \\
\hline
\end{tabular}


Specimen of extended Form of Pellian Equation Table-continued.

\begin{tabular}{|c|c|c|c|c|c|c|c|c|c|}
\hline$a$ & & $y$ & $x$ & $y^{2}-a x^{2}$ & $a$ & & $y$ & $x$ & $y^{2}-a x^{2}$ \\
\hline & (1) & 9 & 1 & +7 & \multirow[t]{5}{*}{79} & 8 & 1 & 0 & +1 \\
\hline . & $(1)$ & 17 & 2 & -7 & & 1 & 8 & 1 & -15 \\
\hline & 1 & 26 & 3 & +10 & & (7) & 9 & 1 & +2 \\
\hline & 16 & 43 & 5 & -1 & & 1 & 71 & 8 & -15 \\
\hline \multirow{5}{*}{75} & & 1 & & & & 16 & 80 & 9 & +1 \\
\hline & 1 & 8 & 1 & $\begin{array}{l}+1 \\
-11\end{array}$ & \multirow[t]{3}{*}{80} & 8 & 1 & 0 & +1 \\
\hline & (1) & 9 & 1 & $\begin{array}{r}11 \\
+\quad 6\end{array}$ & & (1) & 8 & 1 & -16 \\
\hline & 1 & 17 & 2 & -11 & & 16 & 9 & 1 & +1 \\
\hline & 16 & 26 & 3 & +1 & \multirow[t]{3}{*}{82} & 9 & 1 & 0 & +1 \\
\hline \multirow{12}{*}{76} & 8 & 1 & 0 & +1 & & (18) & 9 & 1 & -1 \\
\hline & 1 & 8 & 1 & -12 & & 18 & 163 & 18 & +1 \\
\hline & 2 & 9 & 1 & +5 & \multirow[t]{3}{*}{83} & 9 & 1 & 0 & +1 \\
\hline & 1 & 26 & 3 & -8 & & (9) & 9 & 1 & -2 \\
\hline & 1 & 35 & 4 & +9 & & 18 & 82 & 9 & +1 \\
\hline & 5 & 61 & 7 & -3 & \multirow{3}{*}{84} & 9 & 1 & 0 & +1 \\
\hline & (4) & 340 & 39 & $\begin{array}{r}4 \\
+\quad 4\end{array}$ & & $\begin{array}{c}9 \\
(6)\end{array}$ & $\begin{array}{l}1 \\
9\end{array}$ & $\begin{array}{l}0 \\
1\end{array}$ & $\begin{array}{r}1 \\
-\quad 3\end{array}$ \\
\hline & 5 & $\begin{array}{l}1421 \\
7445\end{array}$ & $\begin{array}{l}163 \\
854\end{array}$ & $\begin{array}{r}3 \\
-\quad 9\end{array}$ & & 18 & 55 & 6 & +1 \\
\hline & 1 & 8866 & 1017 & -8 & \multirow[t]{6}{*}{85} & 9 & 1 & 0 & +1 \\
\hline & 2 & 16311 & 1871 & +5 & & 4 & 9 & 1 & -4 \\
\hline & 1 & 41488 & 4759 & -12 & & (1) & 37 & 4 & +9 \\
\hline & 16 & 57799 & 6630 & +1 & & $\left(\begin{array}{l}1 \\
1\end{array}\right)$ & 46 & 5 & -9 \\
\hline \multirow[t]{8}{*}{77} & 8 & 1 & 0 & +1 & & $\begin{array}{r}4 \\
18\end{array}$ & $\begin{array}{r}83 \\
270\end{array}$ & 9 & +4 \\
\hline & 1 & 8 & 1 & -13 & & 18 & 378 & 41 & -1 \\
\hline & 3 & 9 & 1 & +4 & \multirow[t]{11}{*}{86} & 9 & 1 & 0 & +1 \\
\hline & (2) & 35 & 4 & -7 & & 3 & 9 & 1 & -5 \\
\hline & 3 & 79 & 9 & +4 & & 1 & 28 & 3 & +10 \\
\hline & 1 & 272 & 31 & -13 & & 1 & 37 & 4 & -7 \\
\hline & 16 & 351 & 40 & +1 & & 1 & 65 & 7 & +11 \\
\hline & 8 & & 0 & +1 & & (8) & 102 & 11 & -2 \\
\hline \multirow{5}{*}{78} & 8 & 1 & 0 & +1 & & 1 & 881 & 95 & +11 \\
\hline & 1 & 8 & 1 & -14 & & 1 & 983 & 106 & -7 \\
\hline & (4) & 9 & 1 & +3 & & 1 & 1864 & 201 & +10 \\
\hline & 1 & 44 & 5 & -14 & & 3 & 2847 & 307 & -5 \\
\hline & 16 & 53 & 6 & +1 & & 18 & 10405 & 1122 & +1 \\
\hline
\end{tabular}


Specimen of extended Form of Pellian Equation Table-continued.

\begin{tabular}{|c|c|c|c|c|c|c|c|c|c|}
\hline$a$ & & $y$ & $x$ & $y^{2}-a x^{2}$ & $a$ & & $y$ & $x$ & $y^{2}-a x^{2}$ \\
\hline \multirow[t]{3}{*}{87} & 9 & 1 & 0 & +1 & \multirow[t]{11}{*}{93} & 9 & 1 & 0 & +1 \\
\hline & (3) & 9 & 1 & -6 & & 1 & 9 & 1 & -12 \\
\hline & 18 & 28 & 3 & +1 & & 1 & 10 & 1 & +7 \\
\hline \multirow[t]{7}{*}{88} & 9 & 1 & 0 & +1 & & 1 & 19 & 2 & -11 \\
\hline & 2 & 9 & 1 & -7 & & 4 & 29 & 3 & +4 \\
\hline & 1 & 19 & 2 & +9 & & (6) & 135 & 14 & -3 \\
\hline & (1) & 28 & 3 & -8 & & 4 & 839 & 87 & +4 \\
\hline & 1 & 47 & 5 & +9 & & 1 & 3491 & 362 & -11 \\
\hline & 2 & 75 & 8 & -7 & & 1 & 4330 & 449 & +7 \\
\hline & 18 & 197 & 21 & +1 & & 1 & 7821 & 811 & -12 \\
\hline \multirow{7}{*}{89} & & & & . & & 18 & 12151 & 1260 & +1 \\
\hline & $\begin{array}{l}9 \\
2\end{array}$ & $\begin{array}{l}1 \\
9\end{array}$ & 0 & +1 & \multirow[t]{17}{*}{94} & 9 & 1 & 0 & +1 \\
\hline & 3 & 19 & $\begin{array}{l}1 \\
2\end{array}$ & $\begin{array}{l}-8 \\
+\quad 5\end{array}$ & & 1 & 9 & 1 & -13 \\
\hline & $\left(\begin{array}{l}3 \\
3\end{array}\right)$ & $\begin{array}{l}19 \\
66\end{array}$ & 2 & $\begin{array}{r}+5 \\
5\end{array}$ & & 2 & 10 & 1 & +6 \\
\hline & $\begin{array}{c}3 \\
2\end{array}$ & $\begin{array}{r}60 \\
217\end{array}$ & $\begin{array}{r}7 \\
23\end{array}$ & $\begin{array}{l}-5 \\
+\quad 8\end{array}$ & & 3 & 29 & 3 & -5 \\
\hline & 18 & 500 & $\begin{array}{l}23 \\
53\end{array}$ & +8 & & 1 & 97 & 10 & +9 \\
\hline & & & & -1 & & 1 & 126 & 13 & -10 \\
\hline \multirow[t]{3}{*}{90} & 9 & 1 & 0 & +1 & & 5 & 223 & 23 & +3 \\
\hline & (2) & 9 & 1 & -9 & & 1 & 1241 & 128 & -15 \\
\hline & 18 & 19 & 2 & +1 & & (8) & 1464 & 151 & +2 \\
\hline \multirow[t]{9}{*}{91} & 9 & 1 & 0 & +1 & & 1 & 12953 & 1336 & -15 \\
\hline & 1 & 9 & 1 & -10 & & 5 & 14417 & 1487 & +3 \\
\hline & 1 & 10 & 1 & +9 & & 1 & 85038 & 8771 & -10 \\
\hline & 5 & 19 & 2 & -3 & & 1 & 99455 & 10258 & +9 \\
\hline & (1) & 105 & 11 & +14 & & 3 & 184493 & 19029 & -5 \\
\hline & 5 & 124 & 13 & -3 & & 2 & 652934 & -67345 & +6 \\
\hline & 1 & 725 & 76 & +9 & & 1 & 1490361 & 153719 & -13 \\
\hline & 1 & 849 & 89 & -10 & & 18 & 2143295 & 221064 & +1 \\
\hline & 18 & 1574 & 165 & +1 & \multirow[t]{5}{*}{95} & 9 & 1 & 0 & +1 \\
\hline \multirow[t]{9}{*}{92} & 9 & 1 & 0 & +1 & & 1 & 9 & 1 & -14 \\
\hline & 1 & 9 & 1 & -11 & & $(2)$ & 10 & 1 & +5 \\
\hline & 1 & 10 & 1 & +3 & & 1 & 29 & 3 & -14 \\
\hline & 2 & 19 & 2 & -7 & & 18 & 39 & 4 & +1 \\
\hline & (4) & 48 & 5 & +4 & \multirow[t]{5}{*}{96} & 9 & 1 & 0 & +1 \\
\hline & 2 & 211 & 22 & -7 & & 1 & 9 & 1 & -15 \\
\hline & 1 & 470 & 49 & +3 & & (3) & 10 & 1 & +4 \\
\hline & 1 & 681 & 71 & -11 & & 1 & 39 & 4 & -15 \\
\hline & 18 & 1151 & 120 & +1 & & 18 & 49 & 5 & +1 \\
\hline
\end{tabular}


Specimen of extended Form of Pellian Equation Table-continued,

\begin{tabular}{|c|c|c|c|c|c|c|c|c|c|}
\hline$a$ & & $y$ & $x$ & $y^{2}-a x^{2}$ & $a$ & & $y$ & $x$ & $y^{2}-a x^{2}$ \\
\hline \multirow[t]{12}{*}{97} & 9 & 1 & 0 & +1 & 98 & 9 & 1 & 0 & +1 \\
\hline & 1 & 9 & 1 & -16 & & 1 & 9 & 1 & -17 \\
\hline & 5 & 10 & 1 & +3 & & (8) & 10 & 1 & +2 \\
\hline & 1 & 59 & 6 & -11 & & 1 & 89 & 9 & -17 \\
\hline & 1 & 69 & 7 & +8 & & 18 & 99 & 10 & +1 \\
\hline & (1) & 128 & 13 & -9 & & & & & \\
\hline & (1) & 197 & 20 & +9 & 99 & 9 & 1 & 0 & +1 \\
\hline & 1 & 325 & 33 & -8 & & (1) & 9 & 1 & -18 \\
\hline & 1 & 522 & 53 & +11 & & 18 & 10 & 1 & +1 \\
\hline & 5 & 847 & 86 & -3 & & & & & \\
\hline & 1 & 4757 & 483 & +16 & & & & & \\
\hline & 18 & 5604 & 569 & -1 & & & & & \\
\hline
\end{tabular}

The meaning hardly requires explanation; for each number $a$, we have a series of pairs of increasing numbers, $y, x$, satisfying a series of equations $y^{2}=a x^{2} \pm b$; thus

$$
\begin{array}{rrrr}
a=14 & & \\
y & x & y^{2}-a x^{2} \\
1 & 0 & 1-14.0=1, \\
3 & 1 & 9-14.1=-5, \\
4 & 1 & 16-14.1=+2, \\
11 & 3 & 121-14.9=-5, \\
15 & 4 & 225-14.16=+1 .
\end{array}
$$

The following table, calculated under the superintendence of the Committee, extends from $a=1001$ to $a=1500$ (square numbers omitted); it is (with slight typographical variations) nearly but not exactly in the form of Degen's Table I., the chief difference being that for a number $a$ having a double middle term, or of the form $a^{2}+1$ (such number being further distinguished by an asterisk), the $x, y$ entered in the table are the solutions, not of the equation $y^{2}=a x^{2}+1$, but of the equation $y^{2}=a x^{2}-1$. As remarked above, if we have $y^{2}=a x^{2}-1$, then writing $y_{1}=2 y^{2}+1$ and $x_{1}=2 x y$, we obtain $y_{1}^{2}=a x_{1}^{2}+1$.

Moreover, for each value of $a$, in the first line, the first term, which is the integer part of $\sqrt{a}$, is separated from the other by a semicolon, and the 1 , which is the corresponding first term of the second line, is omitted.

C. XIII. 
The calculations were made by C. E. Bickmore, M.A., of New College, Oxford: his values for $x$ and $y$ have been revised as presently mentioned, but it has been assumed that his values for the periods and subsidiary numbers_(forming the first and second lines of each division of the table) are accurate; in fact, any error therein would cause the resulting values of $x$ and $y$ to be wildly erroneous; but (except in a single instance which was accounted for) the errors in $x$ and $y$ were in every case in a single figure or two or three figures only.

The values of $x$ and $y$ were in every case examined by substitution in the equation $\left(y^{2}=a x^{2}+1\right.$, or $y^{2}=a x^{2}-1$, as the case may be), which should be satisfied by them. These verifications were for the most part made by A. Graham, M.A., of the Observatory, Cambridge. As already mentioned, some errors were detected, and these have been, of course, corrected. The values of $x, y$ given in the table thus satisfy in every case the proper equation $y^{2}=a x^{2}+1$, or $y^{2}=a x^{2}-1$; on the ground above referred to, it is believed that the periods and subsidiary numbers are also accurate.

It may be remarked, in regard to the verification of the equation $y^{2}=a x^{2} \pm 1$ for large values of $x$ and $y$, it is in practice easier and safer to calculate $a x^{2} \pm 1$, and then to compare the square root thereof with the given value of $y$, than to further calculate the value of $y^{2}$. 
The Table 1001 to 1500.

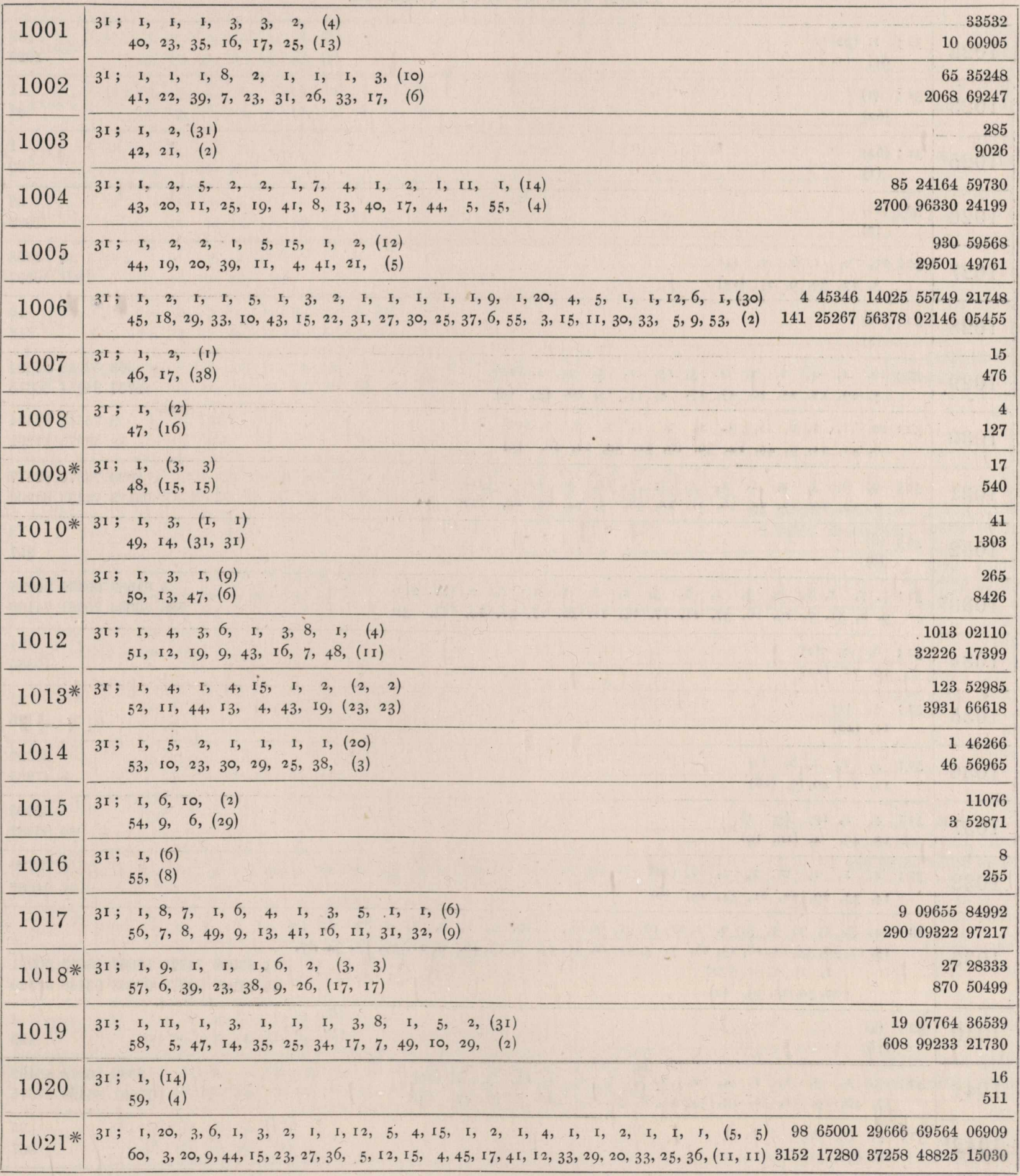


TABLE 1001 To 1500-continued.

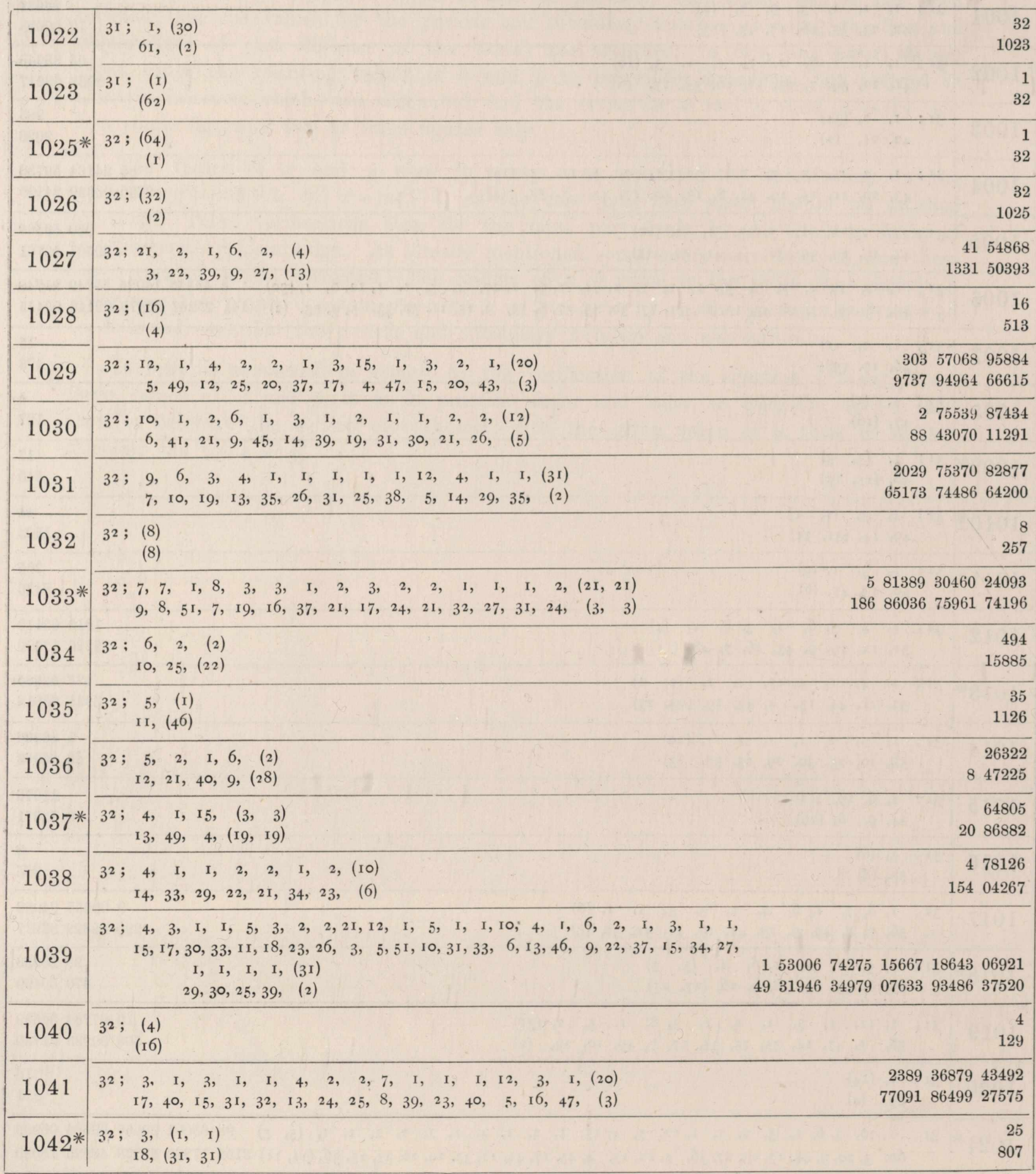


TABLE 1001 To 1500-continued.

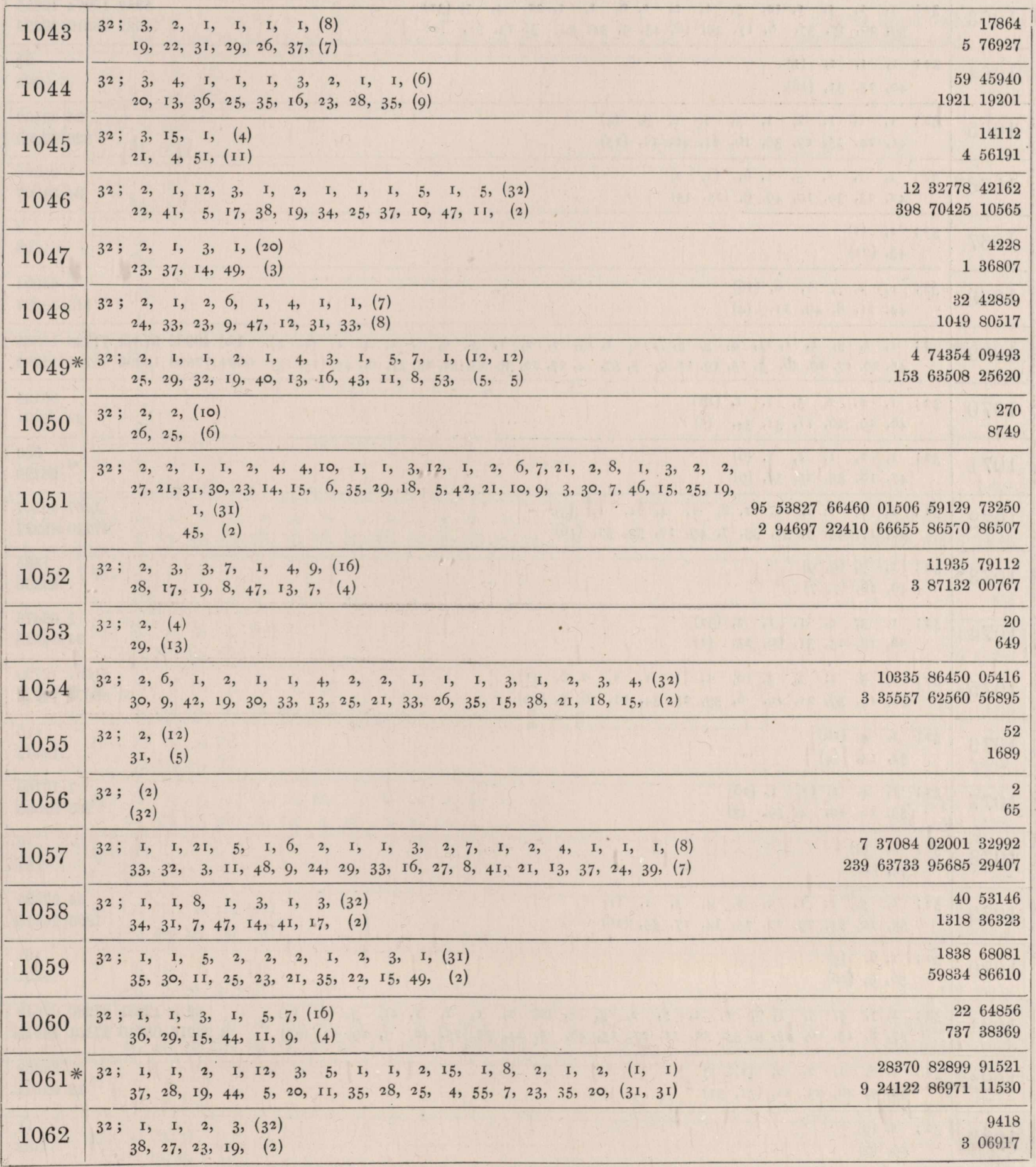


TABLE 1001 To 1500 -continued.

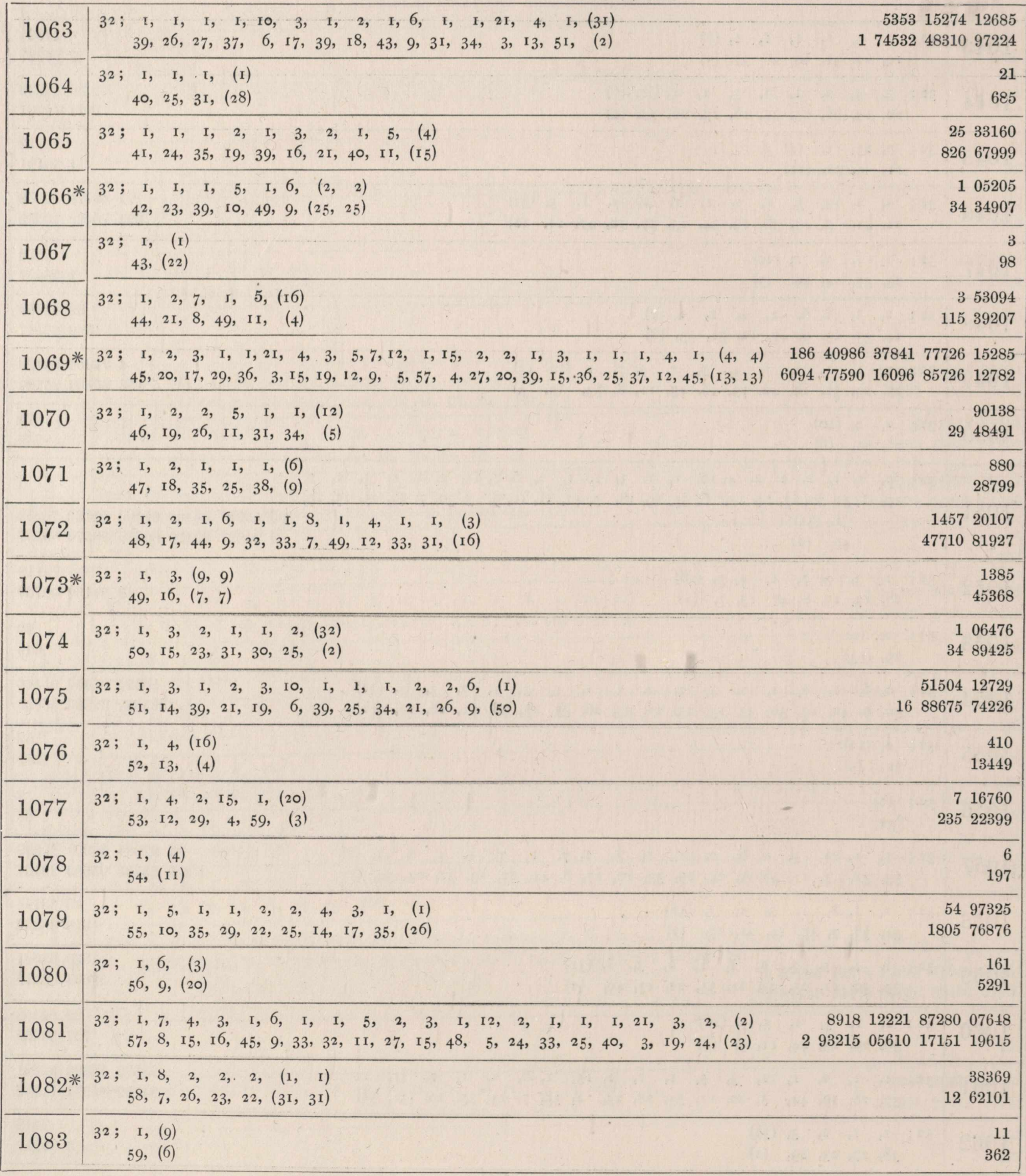


TABLE 1001 To 1500-continued.

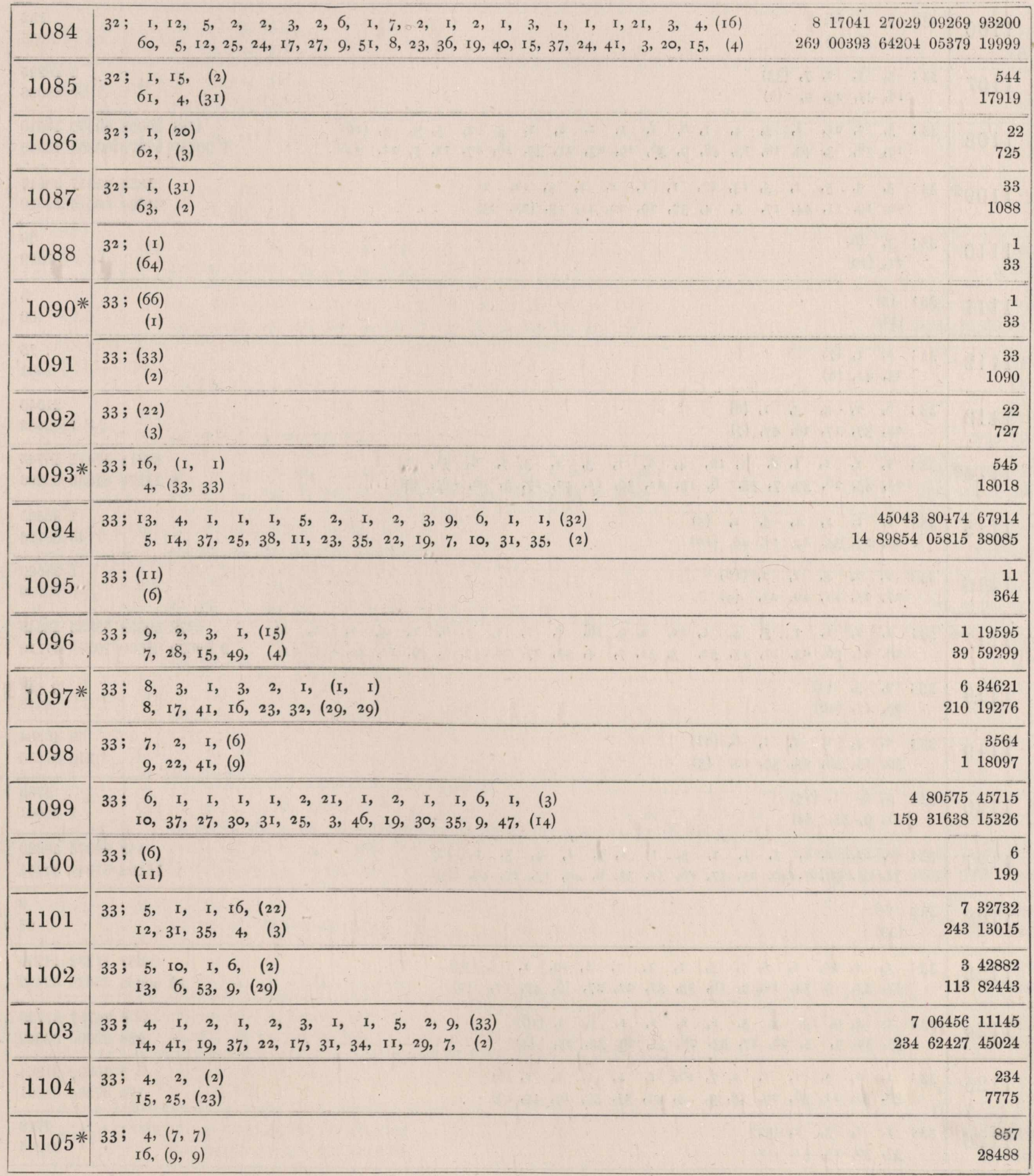


TABLE 1001 To 1500 -continued.

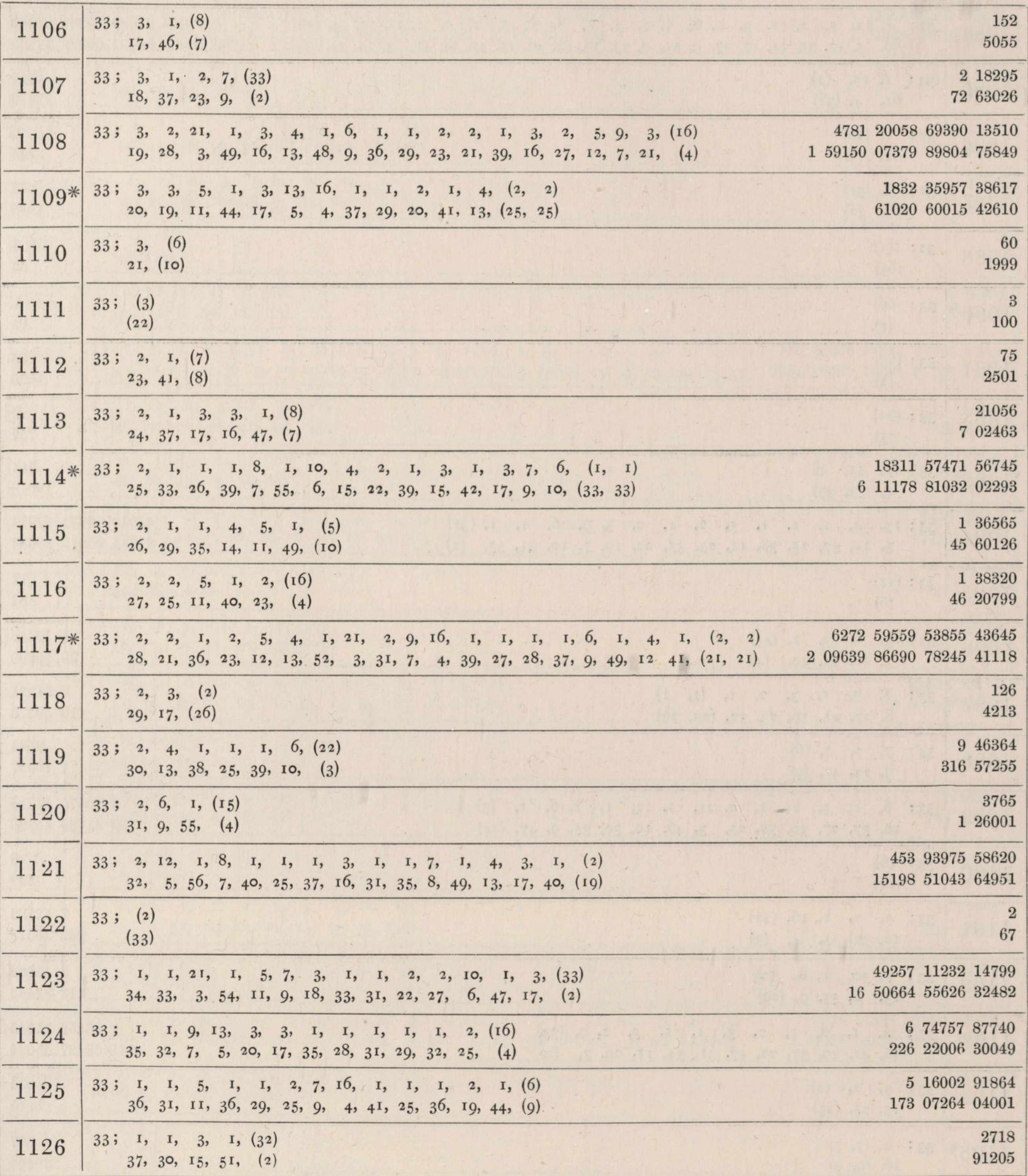


TABLE 1001 To 1500 -continued.

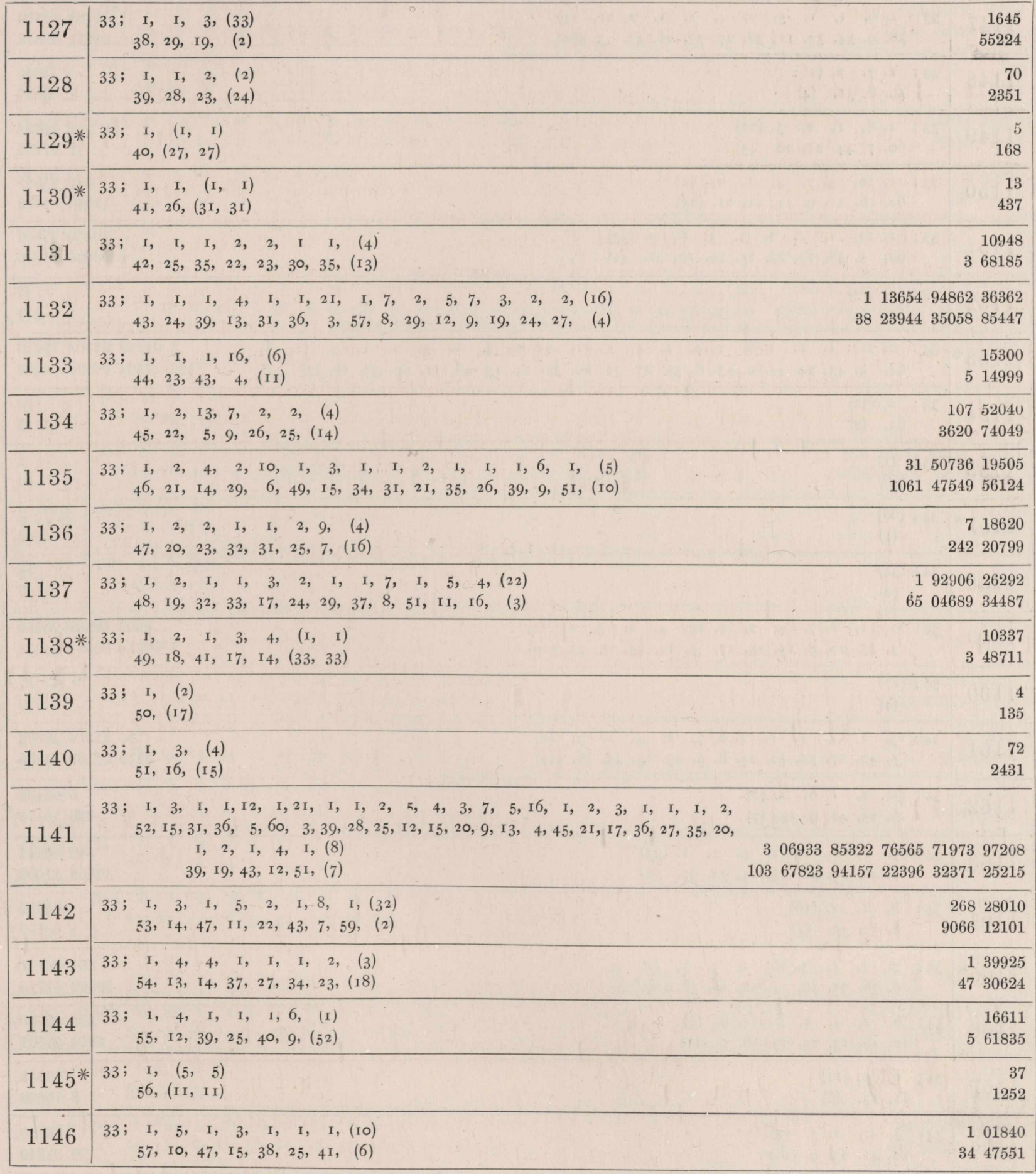

C. XIII. 
TABLE 1001 To 1500-continued.

\begin{tabular}{|c|c|c|}
\hline 1147 & $\begin{array}{r}\text { I, 6, I, I, 5, I, I, I, I, I, 21, (I) } \\
58,9,34,33,1 \mathrm{I}, 38,27,33,26,4 \mathrm{I}, 3,(62)\end{array}$ & $\begin{array}{r}278945403 \\
9447152318\end{array}$ \\
\hline 1148 & $\begin{array}{r}13 ; 7,2,(16) \\
59,8,31, \quad(4)\end{array}$ & $\begin{array}{r}4896 \\
165887\end{array}$ \\
\hline 1149 & $\begin{array}{l}1,8,1,2, \quad 3,(22) \\
60,7,44,21,20, \quad(3)\end{array}$ & $\begin{array}{rr}2 & 12624 \\
72 & 07295\end{array}$ \\
\hline $11 \overline{0} 0$ & $\begin{array}{l}\text { I, } 10,3,7, \quad 4, \quad \mathrm{I}, 2, \quad(2) \\
6 \mathrm{I}, \quad 6,2 \mathrm{I}, 9,14,4 \mathrm{I}, 2 \mathrm{I},(25)\end{array}$ & $\begin{array}{r}34925592 \\
1184384449\end{array}$ \\
\hline 1151 & 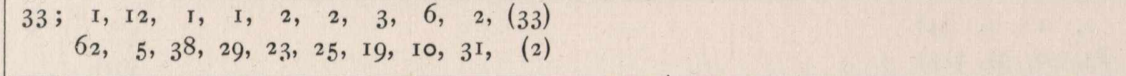 & $\begin{array}{r}1942607807 \\
65905671840\end{array}$ \\
\hline 1152 & $\begin{array}{r}33 ;(15) \\
63,(4) \\
\end{array}$ & $\begin{array}{r}17 \\
577\end{array}$ \\
\hline $1153 *$ & 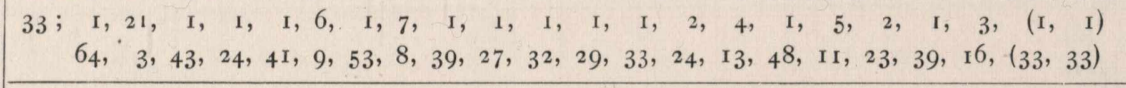 & $\begin{array}{rrrr}3 & 01789 & 02568 & 75073 \\
102 & 47504 & 00230 & 72656\end{array}$ \\
\hline 1154 & $\begin{array}{r}33 ; \quad 1,(32) \\
65,(2)\end{array}$ & $\begin{array}{r}34 \\
1155\end{array}$ \\
\hline 1155 & $33 ; \begin{array}{r}(1) \\
(66)\end{array}$ & $\begin{array}{r}1 \\
34\end{array}$ \\
\hline $1157 *$ & $\begin{array}{r}34 ;(68) \\
(\mathrm{I})\end{array}$ & $\begin{array}{r}1 \\
34\end{array}$ \\
\hline 1158 & $\begin{array}{r}34 ;(34) \\
(2)\end{array}$ & $\begin{array}{r}34 \\
1157\end{array}$ \\
\hline 1159 & 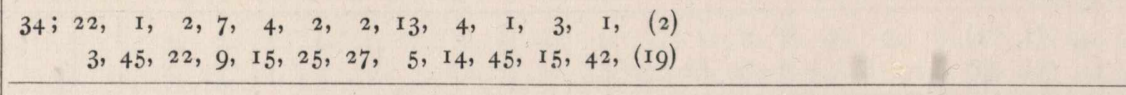 & $\begin{array}{r}49028938575180 \\
1669145551424551\end{array}$ \\
\hline 1160 & $\begin{array}{r}34 ;(17) \\
(4) \\
\end{array}$ & $\begin{array}{r}17 \\
579 \\
\end{array}$ \\
\hline 1161 & 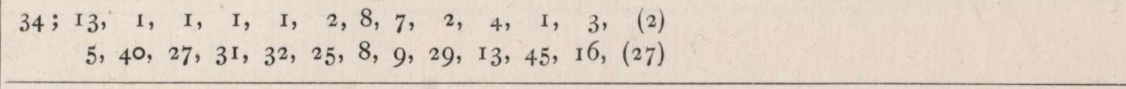 & $\begin{array}{r}56 \quad 21214 \quad 40972 \\
1915 \quad 34168 \quad 54935\end{array}$ \\
\hline 1162 & $\begin{array}{r}34 ; \mathrm{II}, 2, \quad \mathrm{I}, 6, \quad \mathrm{I},(8) \\
6,23,42,9,54,(7)\end{array}$ & $\begin{array}{r}663462 \\
22616173\end{array}$ \\
\hline 1163 & $\begin{array}{c}34, \quad \mathrm{I}, \quad 2, \quad \mathrm{I}, \quad 2, \quad 4, \quad \mathrm{I}, \quad \mathrm{I},(33) \\
7,46, \quad 19,38,23, \\
4\end{array}$ & $\begin{array}{r}36956541 \\
12603 \quad 21002 \\
\end{array}$ \\
\hline 1164 & $\begin{array}{ll}34 ; & 8, \quad \mathrm{I}, \quad \mathrm{I},(\mathrm{r} 6) \\
8,33,35, \quad(4)\end{array}$ & $\begin{array}{r}4930 \\
168199 \\
\end{array}$ \\
\hline $1165^{*}$ & 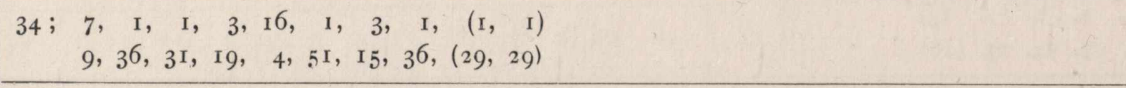 & $\begin{array}{r}86720773 \\
2959961778\end{array}$ \\
\hline 1166 & $\begin{array}{r}6, \quad 1,4, \quad 2, \quad 1, \quad 1,9, \quad(6) \\
10,49, \quad 13,25,29,38,7,(\mathrm{II}) \\
\end{array}$ & $\begin{array}{r}19340870 \\
6604 \quad 27701\end{array}$ \\
\hline 1167 & $\begin{array}{r}64, \quad 5,(\mathrm{II}) \\
\mathrm{II}, \mathrm{I} 3, \quad(6)\end{array}$ & $\begin{array}{r}10943 \\
373828\end{array}$ \\
\hline 1168 & $\begin{array}{r}34 ; \quad 1, \quad 2,7, \quad(4) \\
\text { I } 2,4 \mathrm{I}, 23,9,(\mathrm{I} 6)\end{array}$ & $\begin{array}{r}66750 \\
22 \quad 81249 \\
\end{array}$ \\
\hline
\end{tabular}


TABLE 1001 TO 1500 -continued.

\begin{tabular}{|c|c|c|c|c|}
\hline 1169 & 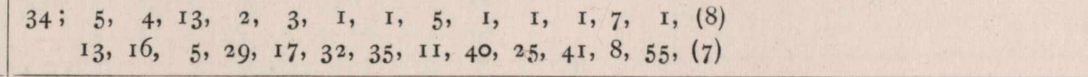 & & $\begin{array}{rr}538 & 84918 \\
18423 & 59949\end{array}$ & 83935 \\
\hline 1170 & $\begin{array}{r}34 ; \quad \mathbf{4},(6) \\
14,49,(9)\end{array}$ & & & $\begin{array}{r}190 \\
6499\end{array}$ \\
\hline 1171 & 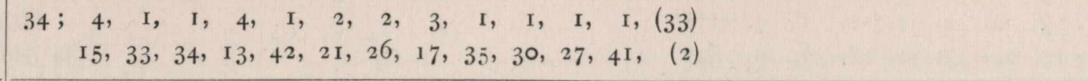 & & $\begin{array}{r}15274 \\
522684\end{array}$ & $\begin{array}{l}30263 \\
76130\end{array}$ \\
\hline 1172 & $\begin{array}{r}4, \quad 3, \quad 1, \quad 3, \quad 1, \quad 1,(16) \\
16, \quad 17,43, \quad 16,31,37, \quad(4)\end{array}$ & & $\begin{array}{r}3 \\
123\end{array}$ & $\begin{array}{l}59890 \\
20649\end{array}$ \\
\hline 1173 & $\begin{array}{r}34 ;(4) \\
(17)\end{array}$ & & & $\begin{array}{r}4 \\
137\end{array}$ \\
\hline 1174 & 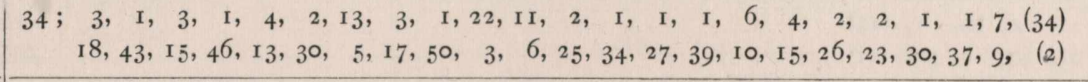 & $\begin{array}{r}1363 \\
467209 \\
4672879\end{array}$ & $\begin{array}{l}1213661406 \\
2065693035\end{array}$ & $\begin{array}{l}74774 \\
91365\end{array}$ \\
\hline 1175 & $\begin{array}{r}34, \quad \mathrm{I}, \quad \mathrm{I}, \quad 2, \quad 5, \quad(\mathrm{I}) \\
\mathrm{I} 9,34,3 \mathrm{I}, 25, \quad \mathrm{II},(50)\end{array}$ & & & $\begin{array}{l}12901 \\
42224\end{array}$ \\
\hline 1176 & $\begin{array}{r}34 ; 3, \quad 2, \quad(2) \\
20,25,(24)\end{array}$ & & & $\begin{array}{r}140 \\
4801\end{array}$ \\
\hline 1177 & 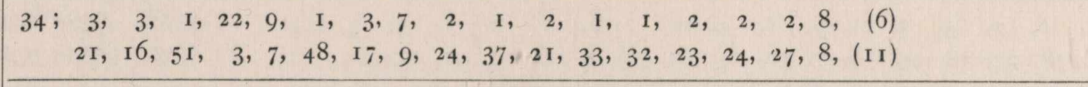 & $\begin{array}{r}276 \\
9482\end{array}$ & $\begin{array}{ll}38354 & 60657 \\
01015 & 39044\end{array}$ & $\begin{array}{l}78460 \\
75351\end{array}$ \\
\hline 1178 & $\begin{array}{r}34,9, \quad(2) \\
22,7,(3 \mathrm{r})\end{array}$ & & & $\begin{array}{r}1736 \\
59583\end{array}$ \\
\hline 1179 & $\begin{array}{r}34 ; \quad \mathrm{I},(33) \\
23,45, \quad(2)\end{array}$ & & & $\begin{array}{r}309 \\
10610\end{array}$ \\
\hline 1180 & $\begin{array}{r}2, \quad \mathrm{I}, \quad 5, \quad \mathrm{I}, \quad \mathrm{I}, \quad 2, \quad \mathrm{I}, \quad(2) \\
24,4 \mathrm{I}, \mathrm{II}, 36,3 \mathrm{I}, 2 \mathrm{I}, 39,(20)\end{array}$ & & 20 & $\begin{array}{l}58950 \\
24999\end{array}$ \\
\hline $1181^{*}$ & 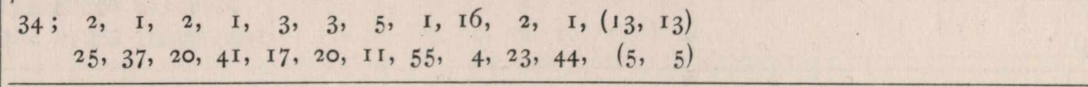 & & $\begin{array}{rr}35 & 62788 \\
1224 & 37647\end{array}$ & $\begin{array}{l}38045 \\
36718\end{array}$ \\
\hline 1182 & $\begin{array}{r}34 ; \quad \text { I, I, I, 2, 2, I, 2, } 1,(\text { (10) } \\
26,33,29,34,23,22,39,19,47,(6)\end{array}$ & & $\begin{array}{r}7 \\
272\end{array}$ & $\begin{array}{l}92682 \\
52587\end{array}$ \\
\hline 1183 & $\begin{array}{r}34 ; \quad 2, \quad 1, \quad 1,7,22, \quad 1, \quad(3) \\
27,29,3^{8}, 9, \quad 3,53,(14)\end{array}$ & & $\begin{array}{r}37 \\
1305\end{array}$ & $\begin{array}{l}96401 \\
76328\end{array}$ \\
\hline 1184 & $\begin{array}{r}34 ; \quad 2, \quad 3, \quad 1,9,\left(1_{7}\right) \\
28,25, \quad 16,49,7, \quad(4)\end{array}$ & i & $\begin{array}{r}7 \\
273\end{array}$ & $\begin{array}{l}95285 \\
65201\end{array}$ \\
\hline 1185 & $\begin{array}{r}34 ; 2, \quad 2, \quad I, \quad 3, \quad I, \quad I, \quad 2, \quad 3, \quad(4) \\
29,21,4 \mathrm{I}, 16,35,3 \mathrm{I}, 24,19,(15)\end{array}$ & & $\begin{array}{r}12 \\
414\end{array}$ & $\begin{array}{l}03200 \\
18751\end{array}$ \\
\hline 1186 & $\begin{array}{r}34 ; \quad 3, \quad 1, \quad 1, \quad 4,(34) \\
30,17,33,34,15, \quad(2)\end{array}$ & & $\begin{array}{r}1 \\
63\end{array}$ & $\begin{array}{l}83522 \\
20195\end{array}$ \\
\hline 1187 & $\begin{array}{r}34 ; \quad 4, \quad \mathrm{I}, 4,9, \quad \mathrm{I}, \mathrm{I}, \quad \mathrm{I}, \quad 2, \quad \mathrm{I},(33) \\
3 \mathrm{I}, \mathrm{I} 3,47, \mathrm{I} 4,7,4 \mathrm{I}, 26,37, \mathrm{I9}, 49,\end{array}$ & & $\begin{array}{r}11347 \\
390951\end{array}$ & $\begin{array}{l}43775 \\
75626\end{array}$ \\
\hline 1188 & $\begin{array}{r}34 ; 2,7,(6) \\
32,9,(\mathrm{II})\end{array}$ & & & $\begin{array}{r}1410 \\
48599\end{array}$ \\
\hline $1189^{*}$ & $\begin{array}{r}34 ; 2, \quad 13,3, \quad 2, \quad 1, \quad 2, \quad 16, \quad 1,6, \quad 1,2, \quad\left(\begin{array}{rr}(1, & 1\end{array}\right) \\
33, \quad 5,20,23,36,25,4,57,9,45,20,(33,33)\end{array}$ & & $\begin{array}{r}2458219 \\
847 \quad 64031\end{array}$ & $\begin{array}{l}58945 \\
17418\end{array}$ \\
\hline
\end{tabular}


TABLE 1001 To 1500-continued.

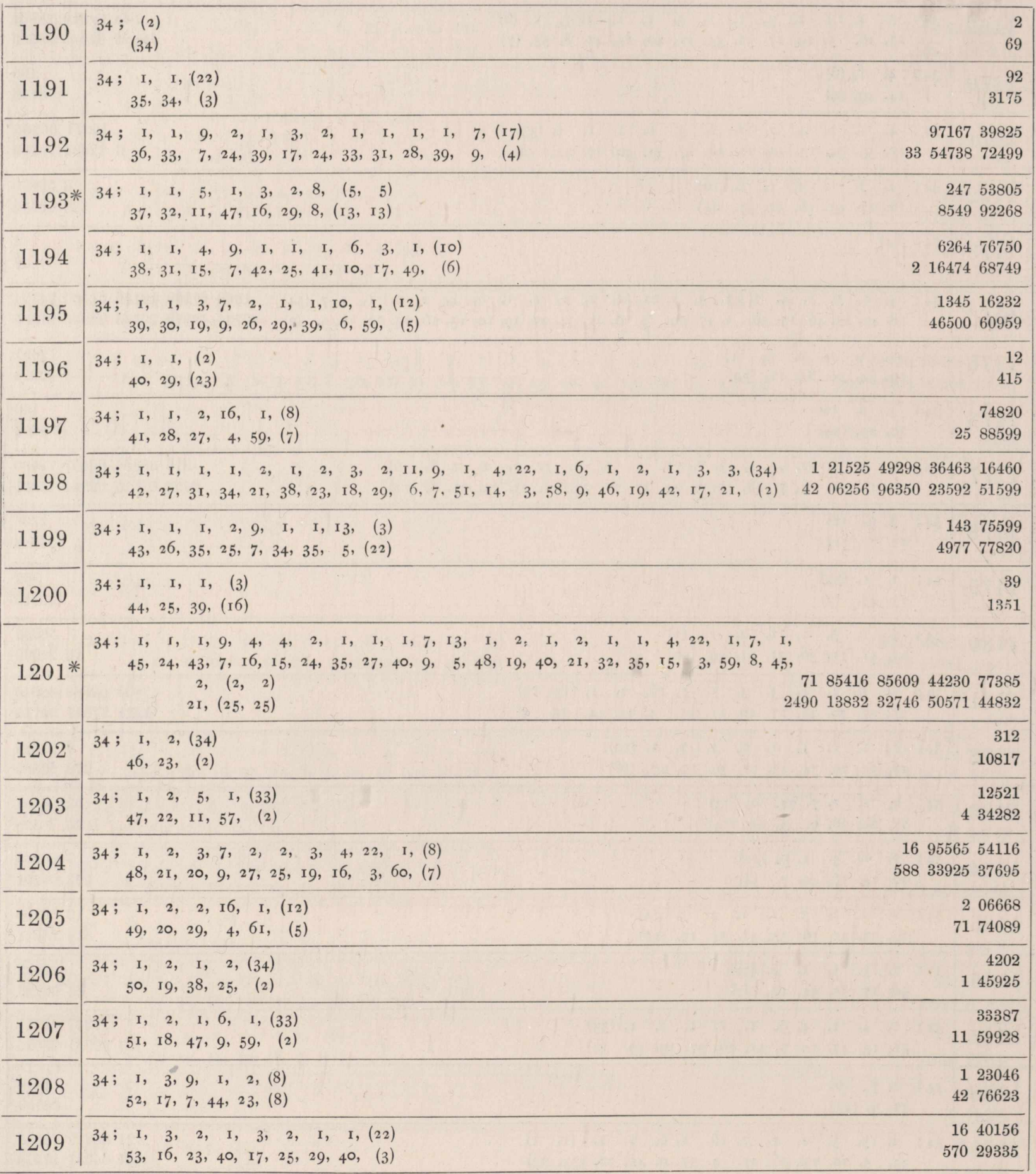


TABLE 1001 To 1500-continued.

\begin{tabular}{|c|c|c|c|}
\hline 1210 & $\begin{array}{r}34, \quad 3, \quad \mathrm{I}, \mathrm{I}, \quad \mathrm{I}, 7, \mathrm{II}, 2, \quad(6) \\
54, \quad 15,39,26,4 \mathrm{I}, 9,6,3 \mathrm{I}, \text { (10) }\end{array}$ & & $\begin{array}{r}43099524 \\
1499219281\end{array}$ \\
\hline 1211 & $\begin{array}{r}34 ; \quad 1,(3) \\
55,(14)\end{array}$ & & $\begin{array}{r}5 \\
174\end{array}$ \\
\hline 1212 & $\begin{array}{r}34,4,2, \quad 1,2,2, \quad \mathrm{I},(\mathrm{r} 6) \\
56,13,24,37,23,2 \mathrm{I}, 47, \quad(4)\end{array}$ & & $\begin{array}{r}365980 \\
12741151\end{array}$ \\
\hline $1213^{*}$ & $\begin{array}{r}34 ; \quad 1,4, \quad 1,4, \quad 1, \quad 1,9,(2, \quad 2) \\
57,12,49, \quad 13,33,36,7,(27,27)\end{array}$ & & $\begin{array}{r}2027117 \\
706 \quad 00734\end{array}$ \\
\hline 1214 & 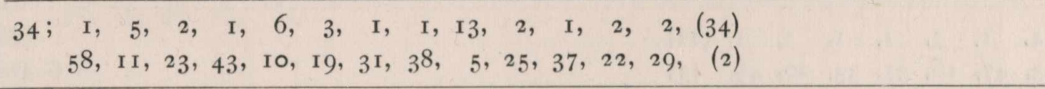 & 209 & $\begin{array}{l}9470260104 \\
0798374975\end{array}$ \\
\hline 1215 & $\begin{array}{r}34 ; \quad(5) \\
59,(10)\end{array}$ & & $\begin{array}{r}7 \\
244\end{array}$ \\
\hline 1216 & $\begin{array}{r}34 ; 6, \quad 1, \quad 3, \quad 4, \quad 2, \quad 1, \quad 1,4,(17) \\
60,9,48, \quad 17, \quad 15,25,31,36,15, \quad(4)\end{array}$ & & $\begin{array}{rr}1916 & 03685 \\
66814 & 48801\end{array}$ \\
\hline $1217^{*}$ & $\begin{array}{r}34 ; \quad 7, \quad \mathrm{I}, 2, \quad \mathrm{I}, 3, \quad \mathrm{I}, \mathrm{I}, \mathrm{I}, \quad(\mathrm{r}, \mathrm{I}) \\
6 \mathrm{I}, 8,47,19,43,16,37,29,32,(3 \mathrm{I}, 3 \mathrm{I})\end{array}$ & & $\begin{array}{rr}7 & 91969 \\
276 & 28256\end{array}$ \\
\hline 1218 & $\begin{array}{r}34 ;(8) \\
62,(7)\end{array}$ & & $\begin{array}{r}10 \\
349\end{array}$ \\
\hline 1219 & 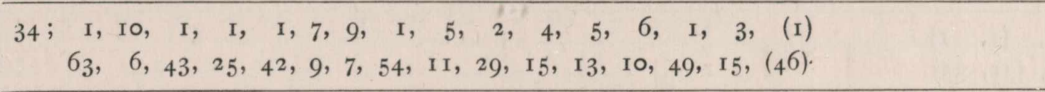 & $\begin{array}{r}76791 \\
2681111\end{array}$ & $\begin{array}{l}4700295135 \\
2454855326\end{array}$ \\
\hline 1220 & $\begin{array}{r}34 ;(12) \\
64, \quad(5)\end{array}$ & & $\begin{array}{r}14 \\
489\end{array}$ \\
\hline 1221 & $\begin{array}{r}34 ; \quad \text { ז, } 16,(2) \\
65,4,(33)\end{array}$ & & $\begin{array}{r}612 \\
21385\end{array}$ \\
\hline 1222 & 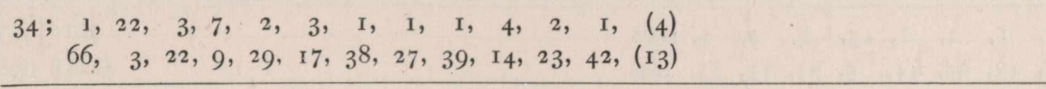 & $\begin{array}{r}22 \\
773\end{array}$ & $\begin{array}{l}1249524122 \\
4245409507\end{array}$ \\
\hline 1223 & $\begin{array}{r}\mathrm{I},(33) \\
67, \quad(2)\end{array}$ & & $\begin{array}{r}35 \\
1224\end{array}$ \\
\hline 1224 & $34 ; \quad(\mathrm{I})$ & & $\begin{array}{r}1 \\
35\end{array}$ \\
\hline $1226^{*}$ & $\begin{array}{r}35 ;(70) \\
(\mathrm{I})\end{array}$ & : & $\begin{array}{r}1 \\
35\end{array}$ \\
\hline 1227 & $35 ;(35)$ & & $\begin{array}{r}35 \\
1226\end{array}$ \\
\hline 1228 & 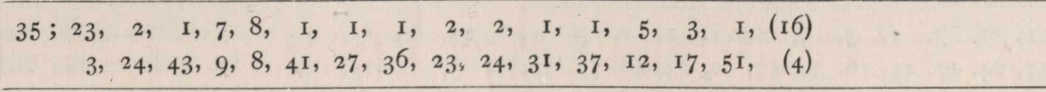 & $\begin{array}{r}44730 \\
15 \quad 67486\end{array}$ & $\begin{array}{l}05971699506 \\
675428 \\
51047\end{array}$ \\
\hline $1229 *$ & $\begin{array}{r}35 ; \quad 17, \quad(1,1) \\
4,(35,35) \\
\end{array}$ & & $\begin{array}{r}613 \\
21490\end{array}$ \\
\hline 1230 & $\begin{array}{r}35 ;(14) \\
(5)\end{array}$ & & $\begin{array}{r}14 \\
491\end{array}$ \\
\hline 1231 & $\begin{array}{r}35 ; \text { II, I, 2, 7, 2, 4, I, I3, 4, I, I, I, I, 6, 2, 2, 4, 3, I, 2, 23, (35) } \\
\quad 6,45,23,9,30,13,54,5, \text { I } 5,37,30,29,39,10,27,26,15,18,39,25,3,\end{array}$ & $\begin{array}{rrr}584 & 79034 & 52350 \\
20517 & 72574 & 24010\end{array}$ & $\begin{array}{l}1522751177 \\
9813487200\end{array}$ \\
\hline
\end{tabular}


TABLE 1001 To 1500-continued.

\begin{tabular}{|c|c|c|c|c|c|}
\hline 1232 & $\begin{array}{r}35 ;(10) \\
(7)\end{array}$ & & & & $\begin{array}{r}10 \\
351\end{array}$ \\
\hline 1233 & $\begin{array}{r}35 ; 8, \quad 1, \quad 3,4,7, \quad 1, \quad 1, \quad 2, \quad 6,9, \quad 1,(6) \\
8,49, \quad 17, \quad 16,9,37,32, \quad 19, \quad 11,7,56,(9)\end{array}$ & & $\begin{array}{r}210 \\
7400\end{array}$ & $\begin{array}{l}76306 \\
75542\end{array}$ & $\begin{array}{l}89984 \\
46657\end{array}$ \\
\hline 1234 & 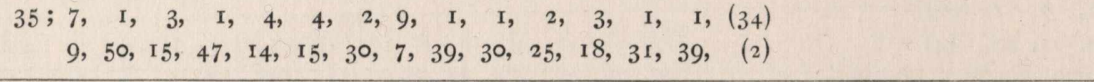 & & $\begin{array}{r}1669 \\
58632\end{array}$ & $\begin{array}{l}10230 \\
78690\end{array}$ & $\begin{array}{l}73856 \\
67265\end{array}$ \\
\hline 1235 & $\begin{array}{r}35 ;(7) \\
(10)\end{array}$ & & & & $\begin{array}{r}7 \\
246\end{array}$ \\
\hline 1236 & 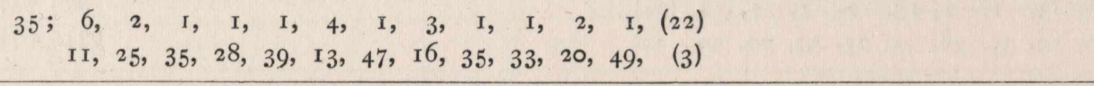 & & & $\begin{array}{l}18261 \\
42027\end{array}$ & $\begin{array}{l}82722 \\
25495\end{array}$ \\
\hline $1237^{*}$ & 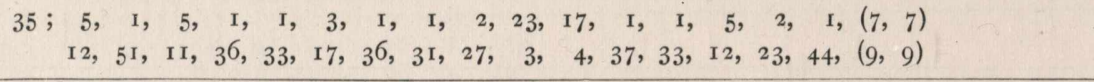 & $\begin{array}{r}30 \\
1083\end{array}$ & 80599 & $\begin{array}{l}95913 \\
40276\end{array}$ & $\begin{array}{l}30265 \\
61982\end{array}$ \\
\hline 1238 & $\begin{array}{r}5, \quad 2, \quad \mathrm{I}, \quad \mathrm{I},(34) \\
\mathrm{I} 3,26, \quad 29,4 \mathrm{I}, \quad(2)\end{array}$ & & & & $\begin{array}{l}25650 \\
02501\end{array}$ \\
\hline 1239 & $\begin{array}{r}35 ;(5) \\
(\mathbf{1} 4)\end{array}$ & & & & $\begin{array}{r}5 \\
176\end{array}$ \\
\hline 1240 & $\begin{array}{r}35 ; \quad \mathrm{I}, \quad 2,7, \quad(2) \\
\mathrm{I} 5,4 \mathrm{I}, 24,9,(3 \mathrm{I})\end{array}$ & & & & $\begin{array}{l}24102 \\
48719\end{array}$ \\
\hline $1241^{*}$ & $\begin{array}{r}35 ; 4, \quad 2, \quad \mathrm{I}, \quad \mathrm{I}, 3,8,(\mathrm{r}, \mathrm{I}) \\
\mathrm{r} 6,25,32,35,19,8,(35,35)\end{array}$ & . & & $\begin{array}{r}9 \\
339\end{array}$ & $\begin{array}{l}65005 \\
95032\end{array}$ \\
\hline 1242 & $\begin{array}{r}35 ; \quad 7, \quad \mathrm{r}, \quad \mathrm{r}, \quad(2) \\
\mathrm{r} 7,9,38,3 \mathrm{r},(23)\end{array}$ & & & & $\begin{array}{l}11780 \\
15151\end{array}$ \\
\hline 1243 & $\begin{array}{r}35 ;, \quad 1,9, \quad(3) \\
18,49,7,(22)\end{array}$ & & & & $\begin{array}{r}4875 \\
71874\end{array}$ \\
\hline 1244 & 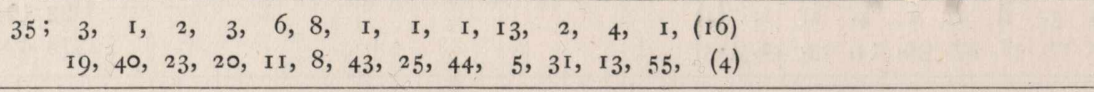 & & $\begin{array}{r}1616 \\
57013\end{array}$ & $\begin{array}{l}45760 \\
08077\end{array}$ & $\begin{array}{l}60360 \\
08799\end{array}$ \\
\hline 1245 & $\begin{array}{r}35 ; \quad \mathrm{I}, \quad \mathrm{I}, \mathrm{I} 7,(\mathrm{I} 4) \\
20,3 \mathrm{I}, 39, \quad 4, \quad(5)\end{array}$ & & & & $\begin{array}{l}13528 \\
34241\end{array}$ \\
\hline 1246 & 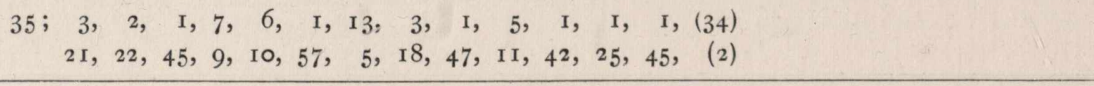 & & $\begin{array}{r}1257 \\
44389\end{array}$ & $\begin{array}{l}54312 \\
66931\end{array}$ & $\begin{array}{l}87288 \\
48735\end{array}$ \\
\hline 1247 & $\begin{array}{r}35, \quad 5,9, \quad(1) \\
22, \quad \mathrm{I} 3,7, \quad(58)\end{array}$ & & & & $\begin{array}{l}26313 \\
29188\end{array}$ \\
\hline 1248 & $\begin{array}{r}35 ; 3,(17) \\
23, \quad(4)\end{array}$ & & & & $\begin{array}{r}159 \\
5617\end{array}$ \\
\hline $1249^{*}$ & $\begin{array}{l}35 ; \quad 2, \mathrm{I}, \mathrm{I} 3,2,7,2, \quad \mathrm{I}, 2,3, \mathrm{I}, 3, \mathrm{I}, \mathrm{I}, \mathrm{I}, 4, \mathrm{I}, 3, \mathrm{I}, 8,23,2,(4,4) \\
24,45,5,32,9,25,37,24, \mathrm{I} 7,45,16,39,27,40, \mathrm{I} 3,48, \mathrm{I} 5,57,8,3,3 \mathrm{I},(\mathrm{I} 5,15)\end{array}$ & $\begin{array}{rr}2 & 61326 \\
92 & 35587\end{array}$ & 40028 & 30963 & $\begin{array}{l}92593 \\
88240\end{array}$ \\
\hline $1250 *$ & $\begin{array}{r}2, \quad \mathrm{I}, \quad 4, \quad 2, \quad \mathrm{I},(\mathrm{I}, \mathrm{I}) \\
25,4 \mathrm{I}, \mathrm{I} 4,25,34,(3 \mathrm{I}, 3 \mathrm{I})\end{array}$ & & & & $\begin{array}{r}7801 \\
75807\end{array}$ \\
\hline 1251 & $\begin{array}{r}35 ; \quad 1, \quad 2, \quad 2, \quad 2, \quad 5, \quad(35) \\
26,37,23,25,27, \quad 13, \quad(2)\end{array}$ & & & $\begin{array}{r}21 \\
775\end{array}$ & $\begin{array}{l}92943 \\
63250\end{array}$ \\
\hline 1252 & 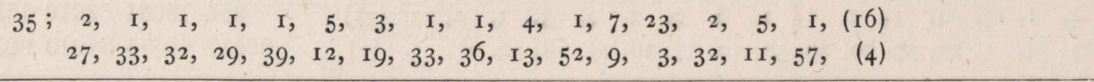 & 32 & $\begin{array}{l}90969 \\
18812\end{array}$ & 00792 & $\begin{array}{l}82080 \\
34849\end{array}$ \\
\hline
\end{tabular}


TABLE 1001 то 1500--continued.

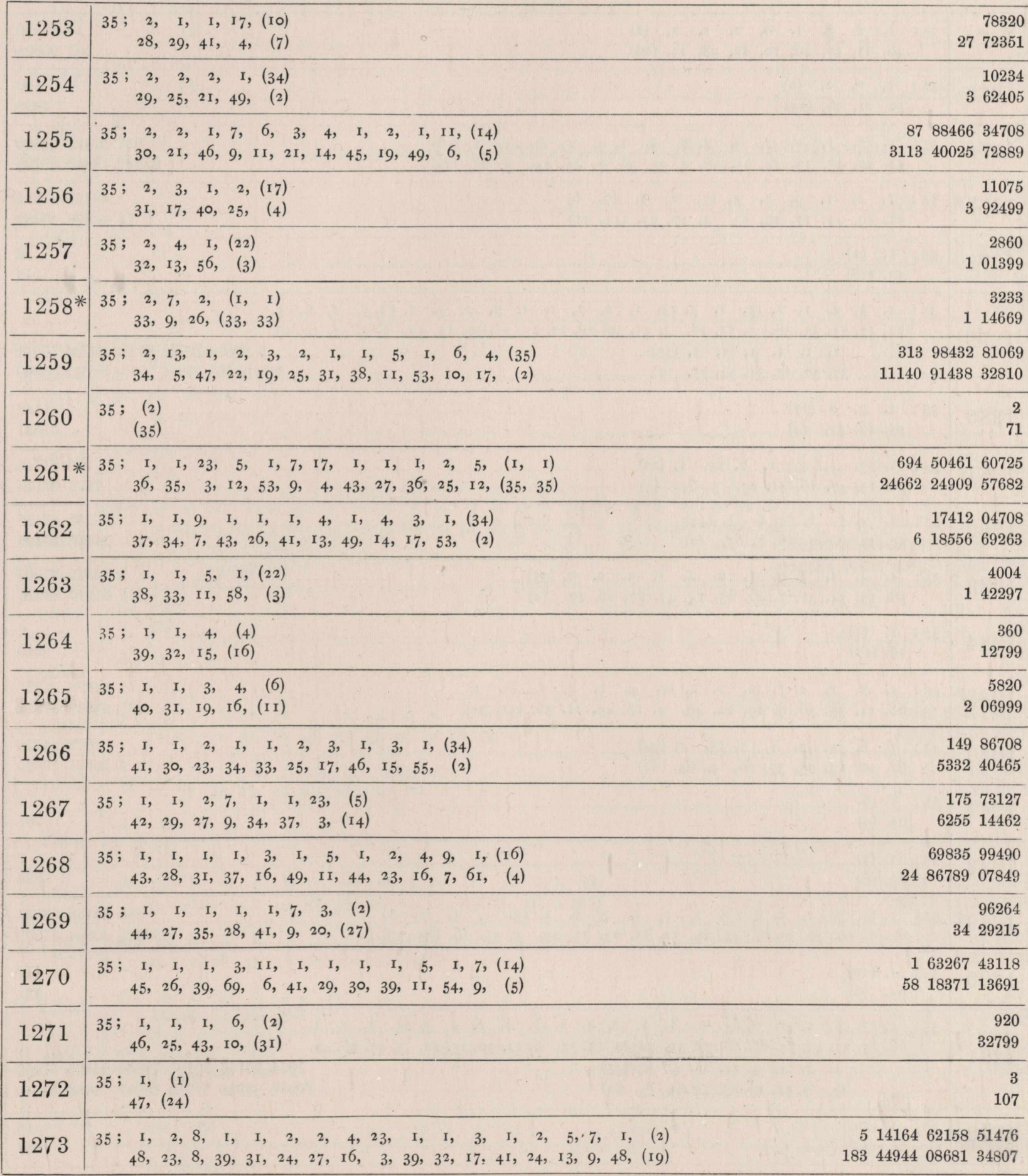


TABLE 1001 TO 1500 -continued.

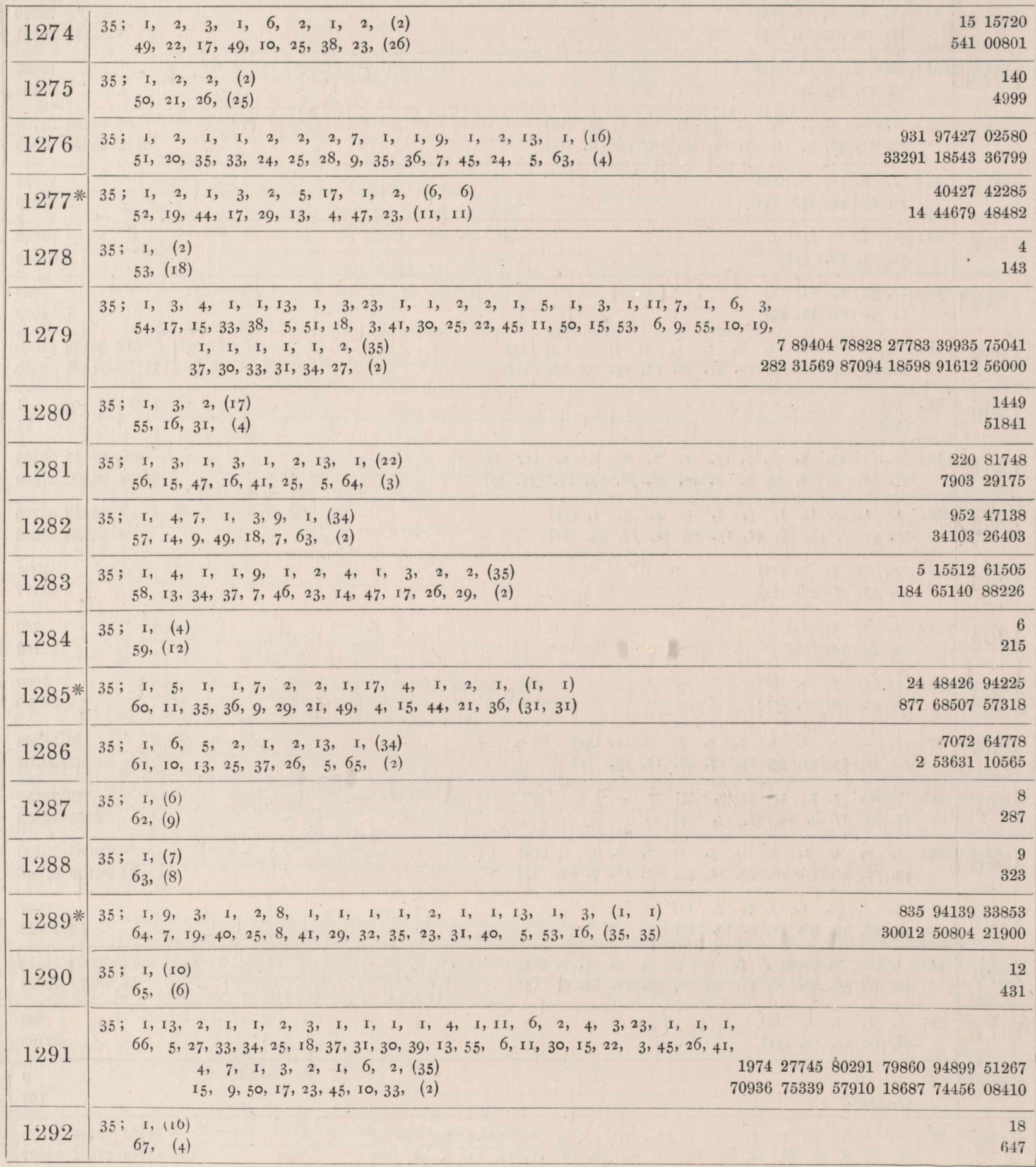


TABLE 1001 то 1500-continued.

\begin{tabular}{|c|c|c|}
\hline 1293 & $\begin{array}{r}35 ; \quad \text { I, } \\
68, \quad(32) \\
68, \quad(3)\end{array}$ & $\begin{array}{r}24 \\
863\end{array}$ \\
\hline 1294 & $\begin{aligned} 35 ; & 1,(34) \\
69, & (2)\end{aligned}$ & $\begin{array}{r}36 \\
1295\end{array}$ \\
\hline 1295 & $35 ; \quad \begin{array}{r}(\mathrm{I}) \\
(70)\end{array}$ & $\begin{array}{r}1 \\
36\end{array}$ \\
\hline $1297^{*}$ & $\begin{array}{r}36 ;(72) \\
\text { (I) }\end{array}$ & $\begin{array}{r}1 \\
36\end{array}$ \\
\hline 1298 & $\begin{array}{r}3^{6} ;(36) \\
(2)\end{array}$ & $\begin{array}{r}36 \\
1.297\end{array}$ \\
\hline 1299 & $\begin{array}{r}36 ;(24) \\
(3) \\
\end{array}$ & $\begin{array}{r}24 \\
865\end{array}$ \\
\hline 1300 & $\begin{array}{r}36 ;(18) \\
(4)\end{array}$ & $\begin{array}{r}18 \\
649\end{array}$ \\
\hline $1301 *$ & $\begin{array}{r}36 ; 14, \quad 2, \quad(2,-2) \\
5,29, \quad(25,25)\end{array}$ & $\begin{array}{r}6025 \\
217318\end{array}$ \\
\hline 1302 & $\begin{array}{r}36 ;(12) \\
(6)\end{array}$ & $\begin{array}{r}12 \\
433\end{array}$ \\
\hline 1303 & 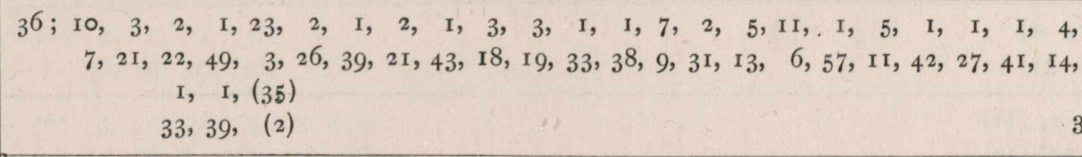 & $\begin{array}{l}\text { 4, } \\
\text { 4, } \\
10127263145189346887 \\
365564742326060871132 \quad 28808\end{array}$ \\
\hline 1304 & $3^{6 ;}\left(\begin{array}{l}(9) \\
(8)\end{array}\right.$ & $\begin{array}{r}9 \\
325\end{array}$ \\
\hline 1305 & $\begin{array}{r}36 ;(8) \\
(9) \\
\end{array}$ & $\begin{array}{r}8 \\
289\end{array}$ \\
\hline $1306^{*}$ & $\begin{array}{r}36 ; 7,4, \quad 1, \quad 2, \text { II, I, } 2, \quad 4,2,9, \quad 1,(7,7) \\
\text { 10, 15, 42, 25, 6, 47, 23, 15, 31, 7, } 58,(9,9)\end{array}$ & $\begin{array}{r}8626815105389 \\
311761210150515\end{array}$ \\
\hline 1307 & $\begin{array}{l}3^{6 ;} 6, \quad \mathrm{I}, \quad \mathrm{I}, 3, \quad \mathrm{I}, \quad 2, \quad \mathrm{I}, \quad \mathrm{I},(35) \\
1 \mathrm{I}, 37,34,17,43,22,3 \mathrm{I}, 4 \mathrm{1}, \quad(2)\end{array}$ & $\begin{array}{r}5414517 \\
195748082\end{array}$ \\
\hline 1308 & $\begin{array}{r}36 ;(6) \\
(12)\end{array}$ & $\begin{array}{r}6 \\
217\end{array}$ \\
\hline 1309 & $\begin{array}{r}36 ; 5, \quad 1, \quad 1,4,3, \quad 1, \quad 1,2,3,17, \quad 1,3, \quad 1,7,(4) \\
13,36,35,15,19,36,33,25,21,4,55,15,52,9,(17)\end{array}$ & $\begin{array}{r}22199221738044 \\
803170174072265\end{array}$ \\
\hline 1310 & $\begin{array}{l}36 ; \quad 6, \quad 2, \quad \mathrm{I}, \quad \mathrm{I}, \quad \mathrm{I}, \quad \mathrm{I}, \quad \mathrm{I},(6) \\
\mathrm{I} 4, \quad \mathrm{II}, 26,35,3 \mathrm{I}, 34,29,4 \mathrm{I}, \text { (Io) }\end{array}$ & $\begin{array}{r}3456382 \\
1251 \quad 00021\end{array}$ \\
\hline 1311 & $\begin{array}{rrrrr}36 ; & 4, & 4 & 2, & (\mathrm{I}) \\
15, & 49, & 14 & 25, & (38) \\
\end{array}$ & $\begin{array}{r}5353 \\
193820\end{array}$ \\
\hline 1312 & $\begin{array}{r}36 ; 4, \quad 1, \quad 1,(17) \\
16,33,39, \quad(4)\end{array}$ & $\begin{array}{r}1467 \\
53137\end{array}$ \\
\hline $1313 *$ & $\begin{array}{rr}(46 & 4 \\
(17, & 17)\end{array}$ & $\begin{array}{r}17 \\
616\end{array}$ \\
\hline
\end{tabular}

C. XIII. 
TABLE 1001 To 1500-continued.

\begin{tabular}{|c|c|c|}
\hline 1314 & $\begin{aligned} & 36 ;(4) \\
&(18)\end{aligned}$ & $\begin{array}{r}4 \\
145\end{array}$ \\
\hline 1315 & $\begin{array}{r}36 ; \quad 1, \quad 4, \quad 11,1,7, \quad(7) \\
19,46,15,6,59,9,(10)\end{array}$ & $\begin{array}{rr}244 & 74351 \\
8875 & 11646\end{array}$ \\
\hline 1316 & $\begin{array}{r}36 ; \quad \mathrm{x}, \quad \mathbf{1}, \quad \mathrm{I}, \quad \mathbf{1}, \quad 2, \quad 3, \quad(2) \\
20,37,31, \quad 32,35,25, \quad 19, \quad(28)\end{array}$ & $\begin{array}{r}65508 \\
2376415\end{array}$ \\
\hline 1317 & 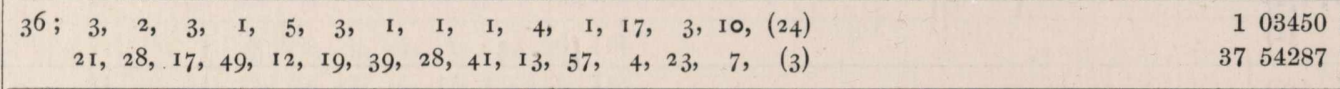 & $\begin{array}{l}9930681500 \\
7077093751\end{array}$ \\
\hline 1318 & 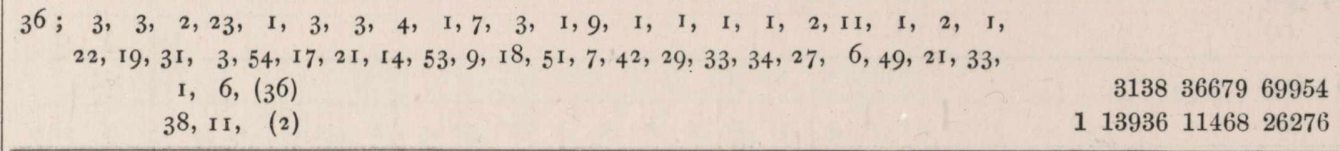 & $\begin{array}{l}0895794554 \\
8176276517\end{array}$ \\
\hline 1319 & $\begin{array}{r}36 ; \quad 6, \quad 1, \quad 13, \quad 1, \quad 1, \quad 1,(35) \\
23, \quad 10,59, \quad 5,46,25,47, \quad(2)\end{array}$ & $\begin{array}{r}33401011 \\
1213059240\end{array}$ \\
\hline 1320 & $3^{6} ;\left(\begin{array}{l}(3) \\
(24)\end{array}\right.$ & $\begin{array}{r}3 \\
109\end{array}$ \\
\hline $1321^{*}$ & $\begin{array}{r}36 ; 2, \mathrm{I}, 8,2,2, \mathrm{I}, \mathrm{I}, 3, \mathrm{I}, 23,2,4,2, \mathrm{I}, 4,6,2, \mathrm{I}, \mathrm{I}, 7,2,(\mathrm{I}, 14) \\
25,45,8,29,24,33,37,16,55,3,32,15,24,43,15,1 \mathrm{I}, 27,3 \mathrm{I}, 40,9,33,(5,5)\end{array}$ & $\begin{array}{l}1961169925 \\
6612796432 \\
\end{array}$ \\
\hline $1322^{*}$ & $\begin{array}{r}36 ; 2, \quad 1,3, \quad \mathrm{I},(\mathrm{I}, \mathrm{I}) \\
26,4 \mathrm{I}, \mathrm{I} 7,38,(3 \mathrm{I}, 3 \mathrm{I}) \\
\end{array}$ & $\begin{array}{r}821 \\
29851\end{array}$ \\
\hline 1323 & $\begin{array}{r}36 ; \quad 2, \quad 1, \quad 2,7, \quad 1, \quad 2, \quad(2) \\
27,37,26,9,47,22,(27)\end{array}$ & $\begin{array}{r}100360 \\
3650401\end{array}$ \\
\hline 1324 & 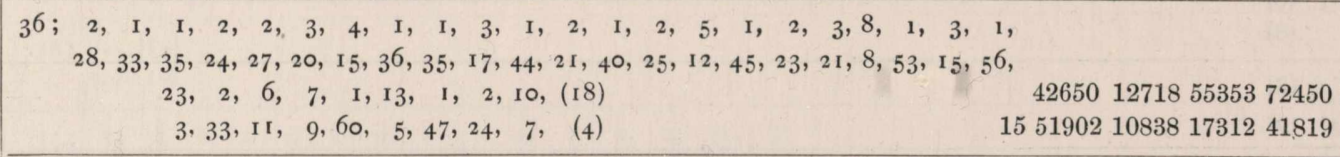 & $\begin{array}{l}3844374810 \\
4098721799\end{array}$ \\
\hline $1325^{*}$ & $\begin{array}{r}36 ;(2, \quad 2) \\
(29,29)\end{array}$ & $\begin{array}{r}5 \\
182\end{array}$ \\
\hline 1326 & $\begin{array}{r}36 ; \quad 2, \quad 2,(2) \\
30,25,(26) \\
\end{array}$ & $\begin{array}{r}70 \\
2549\end{array}$ \\
\hline 1327 & $\begin{array}{r}36 ; 2, \quad 2, \quad \mathrm{I},(35) \\
3 \mathrm{I}, 2 \mathrm{I}, 5 \mathrm{I}, \quad(2)\end{array}$ & $\begin{array}{r}1785 \\
65024\end{array}$ \\
\hline 1328 & $\begin{array}{r}36 ; \quad 2, \quad 3, \quad 1, \quad(3) \\
32, \quad 17,47,(16)\end{array}$ & $\begin{array}{r}369 \\
13447\end{array}$ \\
\hline 1329 & 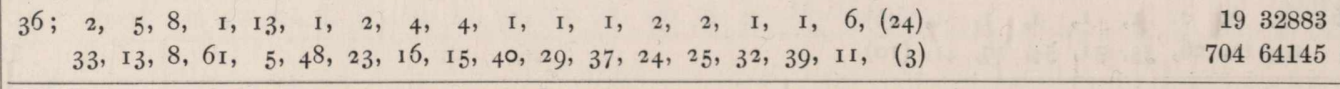 & $\begin{array}{l}5950934116 \\
6388201495\end{array}$ \\
\hline 1330 & $\begin{array}{r}36 ; \quad 2,7, \quad \text { I, I, I, I, } \quad(4) \\
34,9,4 \mathrm{I}, 30,3 \mathrm{I}, 39, \quad(14)\end{array}$ & $\begin{array}{r}34182 \\
1246589\end{array}$ \\
\hline 1331 & 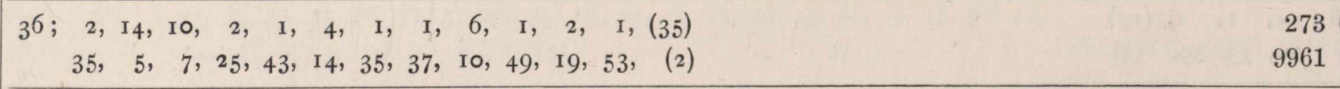 & $\begin{array}{l}0378110727 \\
2037019890\end{array}$ \\
\hline 1332 & $\begin{aligned} 66 ;(2) \\
(36)\end{aligned}$ & $\begin{array}{r}2 \\
73\end{array}$ \\
\hline
\end{tabular}


TABLE 1001 To 1500 -continued.

\begin{tabular}{|c|c|c|}
\hline 1333 & $\begin{array}{r}36 ; \mathrm{r}, \mathrm{I}, 23, \quad \mathrm{I}, 5,7, \quad \mathrm{I}, \mathrm{r} 7,2, \quad \mathrm{r}, \mathrm{1}, \mathrm{I}, 5, \quad(2) \\
37,36,3,59,12,9,6 \mathrm{r}, 4,27,36,29,4 \mathrm{r}, \mathrm{r} 2,(3 \mathrm{I})\end{array}$ & $\begin{array}{rrr}904 & 20166 & 84140 \\
33012 & 64933 & 32151\end{array}$ \\
\hline 1334 & $\begin{array}{r}36 ; \quad 1, \quad 1,9, \quad 1, \quad 13, \quad 1, \quad 2, \quad 2, \quad 1, \quad 1, \quad(2) \\
38,35,7,62, \quad 5,49,22,25,34,35,(23)\end{array}$ & $\begin{array}{rr}864 & 08256 \\
31559 & 72095\end{array}$ \\
\hline 1335 & $\begin{array}{l}36 ; \quad \mathrm{I}, \quad \mathrm{I}, \quad 6, \quad(7) \\
39,34, \mathrm{II},(\mathrm{ro})\end{array}$ & $\begin{array}{r}1235 \\
45124\end{array}$ \\
\hline 1336 & 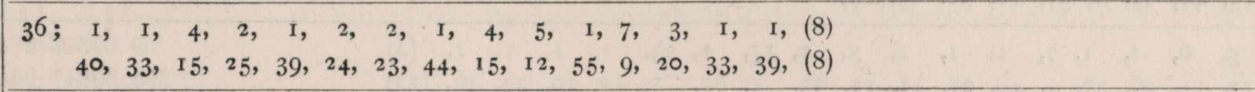 & $\begin{array}{rrr}174 & 56099 & 99622 \\
6380 & 43737 & 19695\end{array}$ \\
\hline 1337 & $\begin{array}{r}36 ; 1, \quad 1,3, \quad 2, \quad 1,8,2,4,(10) \\
41,32,19,23,47,8,31,16, \quad(7)\end{array}$ & $\begin{array}{rr}373 & 97272 \\
13674 & 31647\end{array}$ \\
\hline 1338 & $\begin{array}{r}36 ; \quad 1, \quad 1,2, \quad 1, \quad 2, \text { 10, (12) } \\
42,31,23,39,26, \quad 7, \quad(6)\end{array}$ & $\begin{array}{r}473194 \\
173 \quad 08813\end{array}$ \\
\hline 1339 & $\begin{array}{r}36 ; \quad 1, \quad 1, \quad 2, \quad 4, \quad 1, \quad(4) \\
43,30,27,14,5 \text { I, (13) }\end{array}$ & $\begin{array}{rr}4104 \\
150175\end{array}$ \\
\hline 1340 & $\begin{array}{r}36 ; \quad 1, \quad I, \quad I, \quad I, \quad 6,(18) \\
44,29,31,40,11, \quad(4)\end{array}$ & $\begin{array}{r}19932 \\
729631\end{array}$ \\
\hline 1341 & 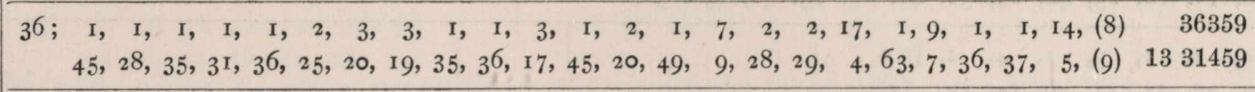 & $\begin{array}{lll}14354 & 86763 & 45320 \\
72360 & 20892 & 39201\end{array}$ \\
\hline 1342 & $\begin{array}{r}36 ; \quad 1, \quad 1, \quad 1, \quad(2) \\
46,27,39,(22)\end{array}$ & $\begin{array}{r}30 \\
1099\end{array}$ \\
\hline 1343 & $\begin{array}{r}36 ; \quad 1, \quad 1, \quad 1, \quad 4, \quad 1,(35) \\
47,26, \quad 43, \quad 13,59, \quad(2)\end{array}$ & $\begin{array}{rr}10591 \\
388128\end{array}$ \\
\hline 1344 & $\begin{array}{r}36 ; \quad 1, \quad 1, \quad 1,(17) \\
48,25,47, \quad(4)\end{array}$ & $\begin{array}{r}165 \\
6049\end{array}$ \\
\hline 1345 & $\begin{array}{r}36 ; \quad 1, \quad 2, \quad(14) \\
49, \quad 24, \quad(5)\end{array}$ & $\begin{array}{r}132 \\
4841\end{array}$ \\
\hline 1346 & $\begin{array}{r}36 ; \quad 1,2, \quad 4, \quad 1,9, \quad 1, \quad 2,(36) \\
50,23,14,55,7,46,25, \quad(2)\end{array}$ & $\begin{array}{rr}92 & 82362 \\
3405 & 50115\end{array}$ \\
\hline 1347 & $\begin{array}{r}36 ; \quad 1, \quad 2, \quad 2, \quad 1, \quad 5, \quad 1,(35) \\
51,22,23,46, \quad 11,61, \quad(2)\end{array}$ & $\begin{array}{rr}1 & 64753 \\
60 & 46682\end{array}$ \\
\hline 1348 & 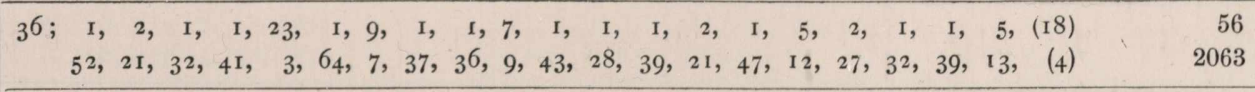 & $\begin{array}{lll}21145 & 67828 & 82376 \\
81035 & 31297 & 13793\end{array}$ \\
\hline 1349 & $\begin{array}{r}36 ; \quad \mathrm{r}, 2, \quad \mathrm{r}, \quad 2, \quad 5, \quad 3, \quad 2, \mathrm{r} 7, \quad \mathrm{r}, \mathrm{I} 3, \quad \mathrm{r}, \quad(2) \\
53,20,4 \mathrm{r}, 25, \mathrm{r} 3,20,3 \mathrm{r}, 4,65, \quad 5,52,(\mathrm{I} 9)\end{array}$ & $\begin{array}{rrr}5 & 55200 & 34840 \\
203 & 91806 & 65951\end{array}$ \\
\hline 1350 & $\begin{array}{r}36 ; \quad 1, \quad 2, \quad 1,7, \quad 2, \quad(2) \\
54, \quad 19,50,9,29,(25)\end{array}$ & $\begin{array}{r}12804 \\
470449\end{array}$ \\
\hline 1351 & $\begin{array}{r}36 ; \quad 1, \quad 3,(10) \\
55, \quad 18, \quad(7)\end{array}$ & $\begin{array}{r}168 \\
6175\end{array}$ \\
\hline 1352 & $\begin{array}{r}36 ; \quad 1, \quad 3,2, \quad 1,(17) \\
56, \quad 17,23,49, \quad(4) \\
\end{array}$ & $\begin{array}{r}3107 \\
114243\end{array}$ \\
\hline 1353 & $\begin{array}{r}3^{6 ;} \mathrm{I}, 3, \quad \mathrm{I}, \mathrm{I}, \mathrm{I}, \mathrm{I}, 2, \quad \mathrm{r}, 8, \quad(2) \\
57, \mathrm{16}, 39,3^{1}, 3^{2}, 37,2 \mathrm{I}, 49,8,(33)\end{array}$ & $\begin{array}{rr}1168536 \\
429 & 82433\end{array}$ \\
\hline
\end{tabular}


TABLE 1001 To 1500 -continued.

\begin{tabular}{|c|c|c|c|}
\hline $1354 *$ & 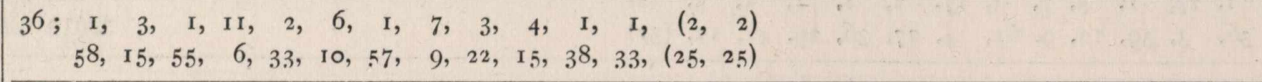 & $\begin{array}{rr}35 & 75033 \\
1315 & 49590\end{array}$ & 94613 \\
\hline 1355 & $\begin{array}{r}36 ; \quad 4, \quad 3, \quad 1, \quad 2, \quad(14) \\
59, \quad 14, \quad 19, \quad 41,26, \quad(5) \\
\end{array}$ & 18 & $\begin{array}{l}49532 \\
23289\end{array}$ \\
\hline 1356 & 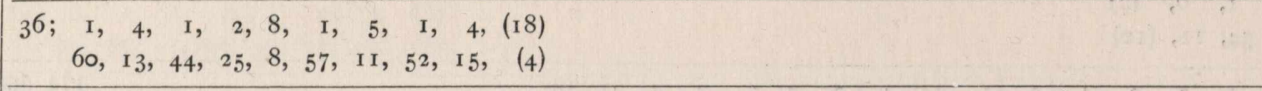 & $\begin{array}{r}5212 \\
191962\end{array}$ & $\begin{array}{l}98370 \\
41799\end{array}$ \\
\hline 1357 & 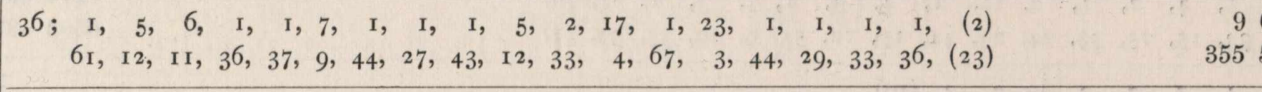 & 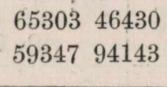 & $\begin{array}{l}64640 \\
72351\end{array}$ \\
\hline 1358 & $\begin{array}{r}36 ; \quad 1, \quad 1,2, \quad 2,(36) \\
62, \quad 11,47,22,31, \quad(2)\end{array}$ & 29 & $\begin{array}{l}81404 \\
99823\end{array}$ \\
\hline 1359 & $\begin{array}{r}36 ; \quad 1, \quad 6, \quad 2, \quad 1, \quad 1,2, \quad 2, \quad 1, \quad 4, \quad 1, \quad 1, \quad(3) \\
63,10,27,34,35,25,23,45,14,37,35,(18)\end{array}$ & $\begin{array}{r}468 \\
17281\end{array}$ & $\begin{array}{l}78231 \\
48040\end{array}$ \\
\hline 1360 & $\begin{array}{r}36,7, \quad 4, \quad 1, \quad(3) \\
64,9, \quad 15,49,(16) \\
\end{array}$ & 2 & $\begin{array}{r}7749 \\
85769\end{array}$ \\
\hline $1361^{*}$ & 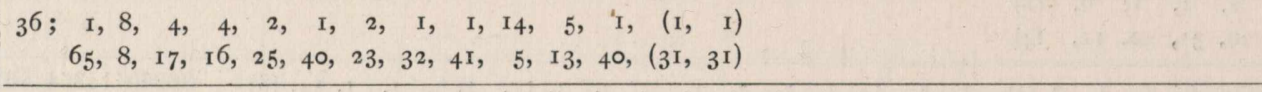 & $\begin{array}{r}3593384 \\
132566186\end{array}$ & $\begin{array}{l}58529 \\
45260\end{array}$ \\
\hline 1362 & $\begin{array}{r}36 ; \quad 1,9, \quad 1, \quad 1,3, \quad 1,4, \quad 2,(36) \\
66,7,39,34,17,49,14,33, \quad(2) \\
\end{array}$ & $\begin{array}{r}371 \\
13701\end{array}$ & $\begin{array}{l}27048 \\
84257\end{array}$ \\
\hline 1363 & $\begin{array}{r}36, \quad 11,3,7, \quad(1) \\
67, \quad 6, \quad 23,9,(58)\end{array}$ & 34 & $\begin{array}{l}93495 \\
51726\end{array}$ \\
\hline 1364 & $\begin{array}{r}36 ; \mathrm{I}, \mathrm{I} 3, \mathrm{I}, 3, \quad \mathrm{r}, 2, \quad(6) \\
68,5,55, \mathrm{r} 6,43,25,(\mathrm{II})\end{array}$ & $\begin{array}{r}2 \\
106\end{array}$ & $\begin{array}{l}87730 \\
26551\end{array}$ \\
\hline 1365 & $\begin{array}{r}36 ; \quad 17, \quad(2) \\
69, \quad 4, \quad(35) \\
\end{array}$ & . & $\begin{array}{r}684 \\
25271\end{array}$ \\
\hline 1366 & 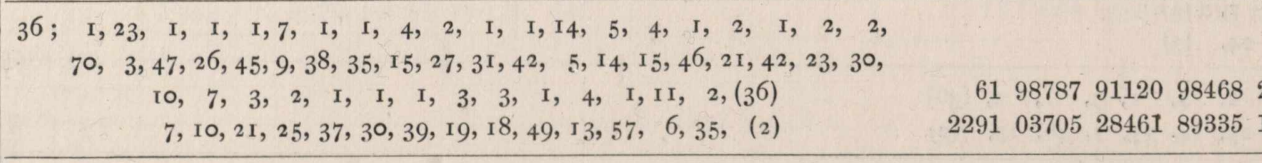 & $\begin{array}{l}2312864853 \\
14238 \\
95408\end{array}$ & $\begin{array}{l}64042 \\
99525\end{array}$ \\
\hline 1367 & $\begin{array}{r}36 ; \quad, \quad(35) \\
7 \mathrm{I}, \quad(2) \\
\end{array}$ & i & $\begin{array}{r}37 \\
1368\end{array}$ \\
\hline 1368 & $\begin{array}{r}36 ;(1) \\
(72) \\
\end{array}$ & 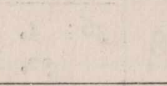 & $\begin{array}{r}1 \\
37\end{array}$ \\
\hline $1370 *$ & $\begin{array}{r}37 ;(74) \\
(\mathrm{I})\end{array}$ & . & $\begin{array}{r}1 \\
37\end{array}$ \\
\hline 1371 & $\begin{array}{r}37 ;(37) \\
(2)\end{array}$ & . & $\begin{array}{r}37 \\
1370\end{array}$ \\
\hline 1372 & 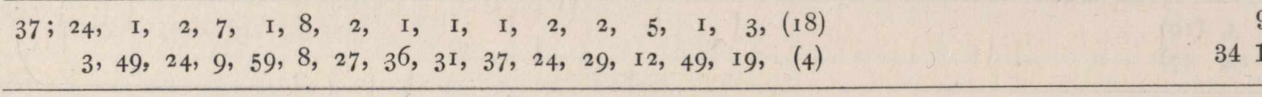 & 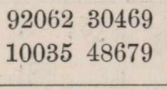 & $\begin{array}{l}34552 \\
27167\end{array}$ \\
\hline $1373^{*}$ & $\begin{array}{r}37 ; 18, \quad(1, \quad 1) \\
4,(37,37)\end{array}$ & 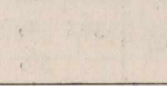 & $\begin{array}{r}685 \\
25382\end{array}$ \\
\hline 1374 & 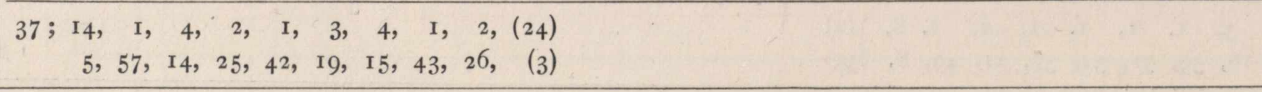 & $\begin{array}{r}41422 \\
15 \quad 35443\end{array}$ & $\begin{array}{l}89074 \\
25045\end{array}$ \\
\hline
\end{tabular}


TABLE 1001 To 1500 -continued.

\begin{tabular}{|c|c|}
\hline 1375 & $\begin{array}{r}37 ; \mathrm{I} 2, \quad 2, \quad \mathrm{I}, 7, \quad \mathrm{I}, \mathrm{I}, \quad 3, \quad 2, \quad \mathrm{r}, 2, \quad(6) \\
6,25,46,9,39,34,19,25,39,26,(\mathrm{II})\end{array}$ \\
\hline 1376 & $\begin{array}{r}37 ; \mathrm{ro}, \mathrm{I}, \mathrm{I}, 2, \quad 2, \quad 3, \quad \mathrm{I},(17) \\
7,4 \mathrm{I}, 32,25,28, \quad 17,55, \quad(4)\end{array}$ \\
\hline 1377 & $\begin{array}{r}1112162480 \\
41270070401\end{array}$ \\
\hline $1378^{*}$ & $\begin{array}{r}37 ; 8,(4,4) \\
9,(\mathrm{r} 7, \mathrm{r} 7)\end{array}$ \\
\hline 1379 & $\begin{array}{r}37 ; 7,2,2,2, \quad 1, \quad 1,3,3,(10) \\
10,29,26,25,34,37,19,22,(7)\end{array}$ \\
\hline 1380 & $\begin{array}{r}37 ; \quad \mathrm{I},(2) \\
\mathrm{II}, 49,(20)\end{array}$ \\
\hline $1381 *$ & 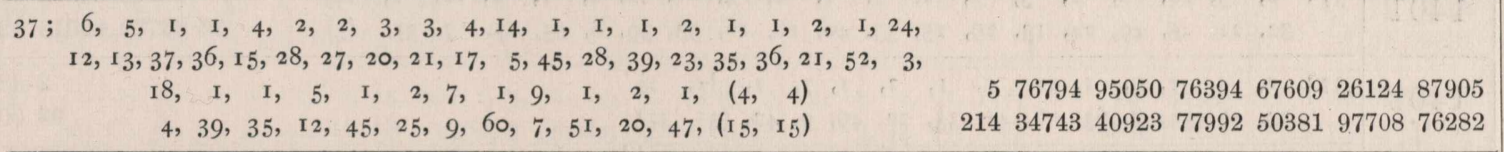 \\
\hline 1382 & $\begin{array}{r}57, \quad \mathrm{r}, 2,2, \quad \mathrm{I}, \quad \mathrm{r},(36) \\
\mathrm{I} 3,46,23,26,31,43, \quad(2)\end{array}$ \\
\hline 1383 & $\begin{array}{r}5, \quad 3, \quad 2, \quad \mathrm{r},(\mathrm{II}) \\
\mathrm{I} 4,2 \mathrm{I}, 23,49, \quad(6)\end{array}$ \\
\hline 1384 & $\begin{array}{r}47, \quad \mathrm{I},(\mathrm{I} 7) \\
\mathrm{I} 5,57, \quad(4)\end{array}$ \\
\hline $1385^{*}$ & $\begin{array}{r}47, \quad \mathrm{r}, \mathrm{I}, \quad \mathrm{I}, \quad(3,3) \\
\mathrm{r} 6,4 \mathrm{I}, 29,40,(\mathrm{r} 9, \mathrm{I} 9)\end{array}$ \\
\hline 1386 & $\begin{array}{r}47,2, \quad 1, \quad(2) \\
17,25,41,(22) \\
\end{array}$ \\
\hline 1387 & $\begin{array}{r}37 ; \\
18,9,(37) \\
18, \quad(2)\end{array}$ \\
\hline 1388 & $\begin{array}{l}37 ; \quad 1,9, \quad 1,8, \quad 2, \quad 2, \quad 5, \quad 3,(18) \\
19,52,7,61,8,29,28, \quad 13,23, \quad(4)\end{array}$ \\
\hline 1389 & $\begin{array}{r}37 ; \quad 1, \quad 2, \quad 2,(24) \\
20,43,23,31, \quad(3)\end{array}$ \\
\hline 1390 & 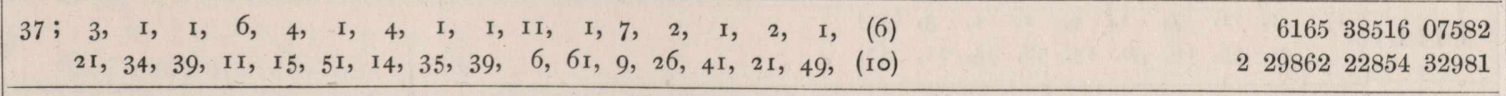 \\
\hline 1391 & $\begin{array}{r}37 ; 3, \quad 2, \quad 1, \quad 1, \quad 1, \quad 6, \quad 1, \quad(4) \\
22,25,38,29,43,10,55,(13)\end{array}$ \\
\hline 1392 & $\begin{array}{r}37 ; \quad(4) \\
23,(\mathrm{r} 6)\end{array}$ \\
\hline 1393 & $\begin{array}{r}37 ; \quad(10) \\
24, \quad(7) \\
\end{array}$ \\
\hline 1394 & $\begin{array}{r}37 ; \quad 2, \quad 1,(36) \\
25,49, \quad(2)\end{array}$ \\
\hline
\end{tabular}


TABLE 1001 To 1500-continued.

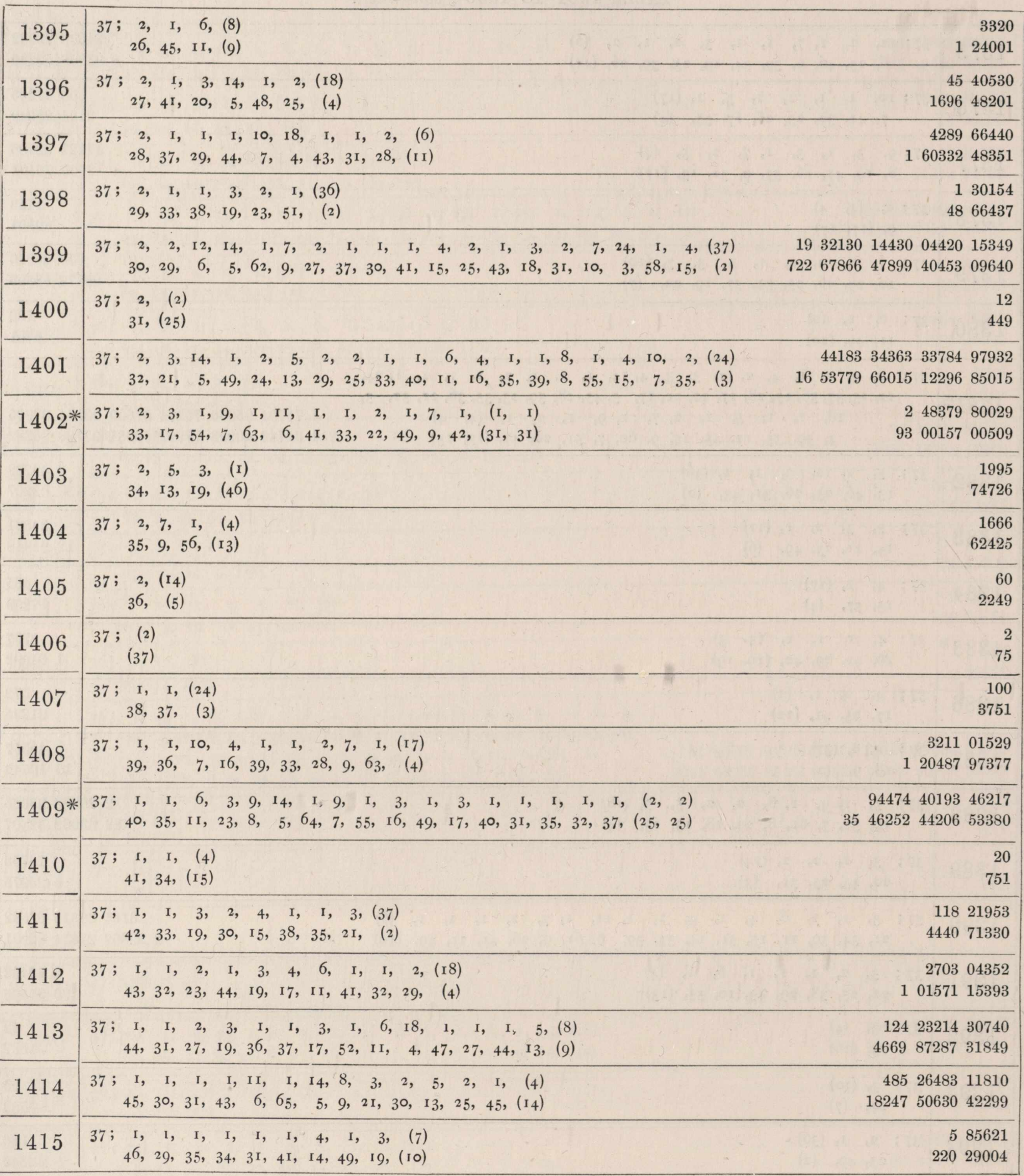


TABLE 1001 to 1500-continued.

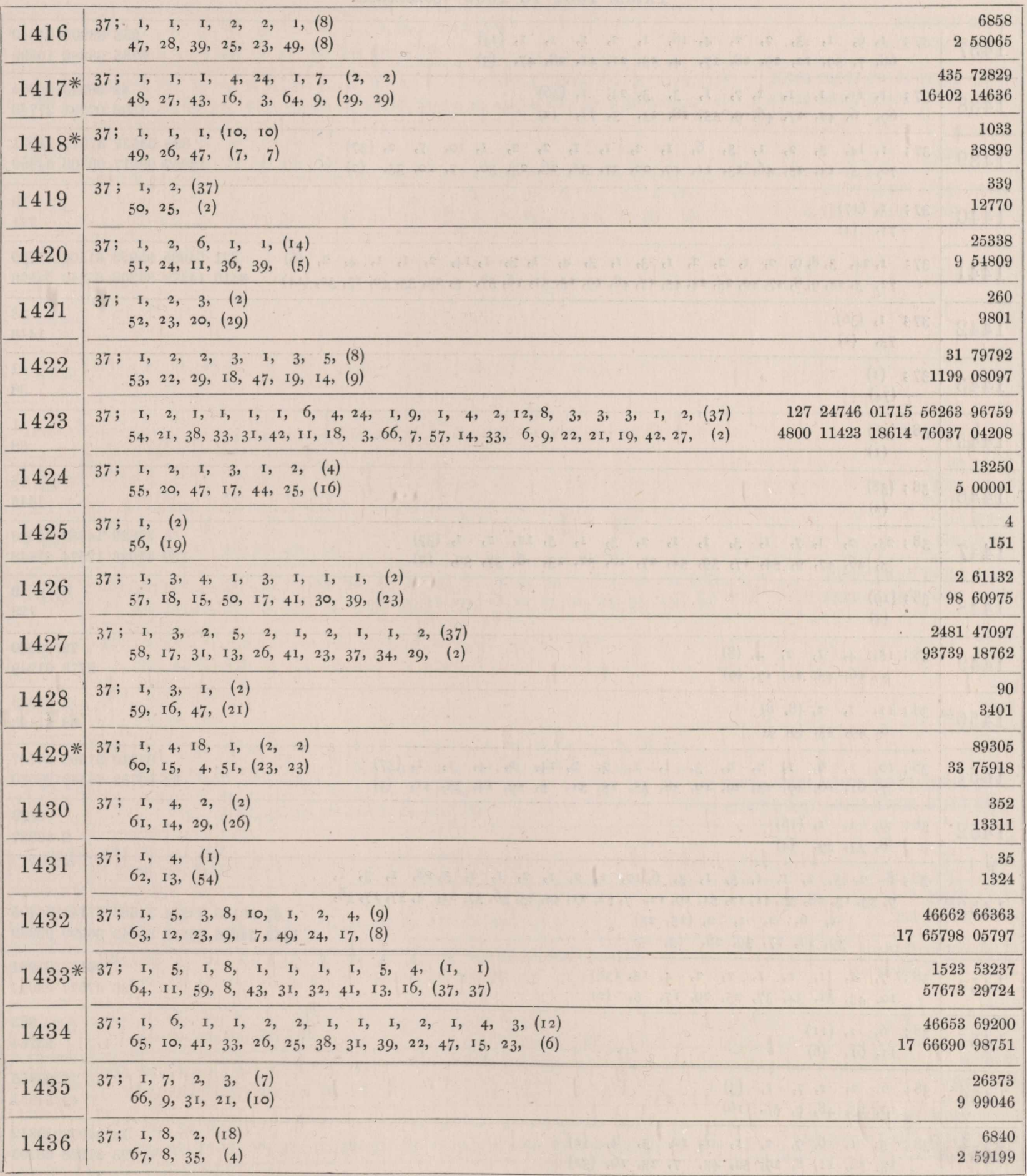


TABLE 1001 TO 1500 -continued.

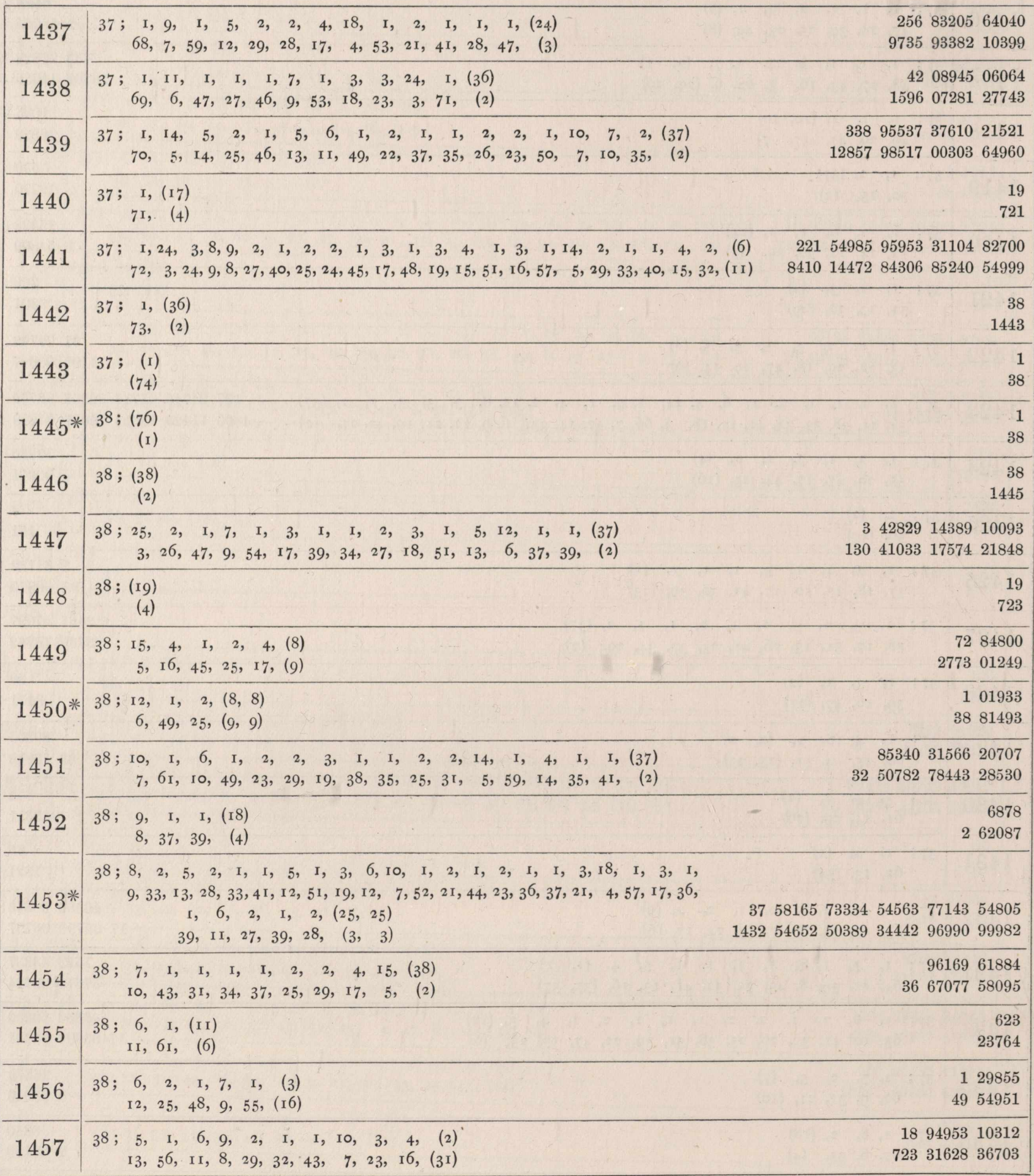


TABLE 1001 To 1500-continued.

\begin{tabular}{|c|c|c|c|c|}
\hline 1458 & 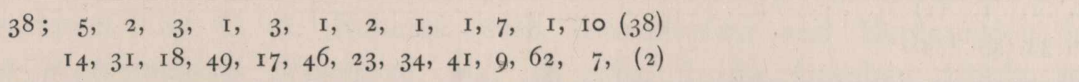 & & $\begin{array}{rr}78 & 88890 \\
3012 & 27540\end{array}$ & 25540 \\
\hline 1459 & 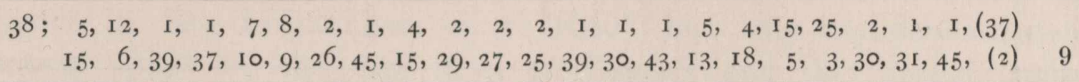 & $\begin{array}{r}2569424820 \\
8143956337\end{array}$ & $\begin{array}{l}9812422872 \\
9940345032\end{array}$ & 52281 \\
\hline 1460 & $\begin{array}{l}38 ; 4, \quad \mathrm{I}, 3, \quad 4, \quad \mathrm{I}, \mathrm{I},(\mathrm{I} 8) \\
16,49, \mathrm{1} 9, \mathrm{1} 6,35,4 \mathrm{I}, \quad(4)\end{array}$ & & & 25898 \\
\hline 1461 & 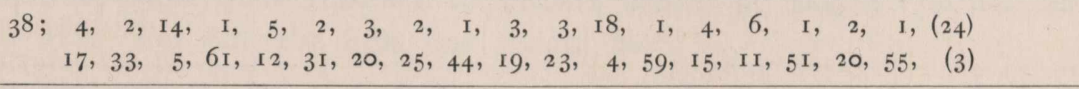 & $\begin{array}{rr}10897 \\
416529\end{array}$ & $\begin{array}{l}3350665845 \\
1619797289\end{array}$ & 02228 \\
\hline 1462 & $\begin{array}{r}38 ; \quad 4, \quad(4) \\
18,(17)\end{array}$ & & & $\begin{array}{r}72 \\
2753\end{array}$ \\
\hline 1463 & $\begin{array}{r}3^{8} ;(4) \\
(19)\end{array}$ & & & $\begin{array}{r}4 \\
153\end{array}$ \\
\hline 1464 & $\begin{array}{r}38 ; \quad 1, \quad 4, \quad 2, \quad 1, \quad(5) \\
20,49, \quad 15,25,47,(12)\end{array}$ & & & $\begin{array}{l}23729 \\
07925\end{array}$ \\
\hline $1465^{*}$ & 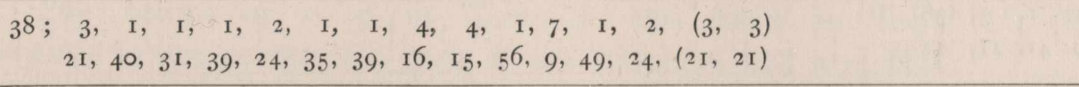 & & $\begin{array}{rr}2 & 14427 \\
82 & 07269\end{array}$ & $\begin{array}{l}21285 \\
84932\end{array}$ \\
\hline $1466^{*}$ & $\begin{array}{r}38 ; 3,2, \quad 7,4, \quad 2, \quad \mathrm{I},(2, \quad 2) \\
22,3 \mathrm{I}, 10, \quad 17,26,4 \mathrm{I},(25,25)\end{array}$ & & $\begin{array}{r}40 \\
1533\end{array}$ & 05185 \\
\hline 1467 & $\begin{array}{r}38 ; 3, \quad 3, \quad 6, \quad 1, \quad \text { I, } 1,(37) \\
23,22, \quad 11,46,27,49, \quad(2)\end{array}$ & & $\begin{array}{r}16 \\
640\end{array}$ & $\begin{array}{l}73045 \\
80026\end{array}$ \\
\hline 1468 & 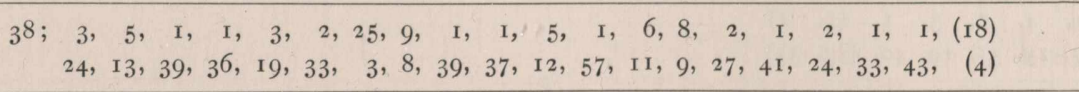 & $\begin{array}{rr}18883 \\
723520\end{array}$ & $\begin{array}{l}7303664922 \\
4628134792\end{array}$ & 35216 \\
\hline 1469 & $\begin{array}{r}38 ; \quad \begin{array}{r}3 \\
3\end{array}, \quad \mathrm{i}, \quad(4) \\
25, \quad 4,61,(13)\end{array}$ & & & $\begin{array}{l}19836 \\
60265\end{array}$ \\
\hline 1470 & $\begin{array}{r}38 ; \quad 2, \quad 1,(14) \\
26,49, \quad(5)\end{array}$ & & & $\begin{array}{r}138 \\
5291\end{array}$ \\
\hline 1471 & 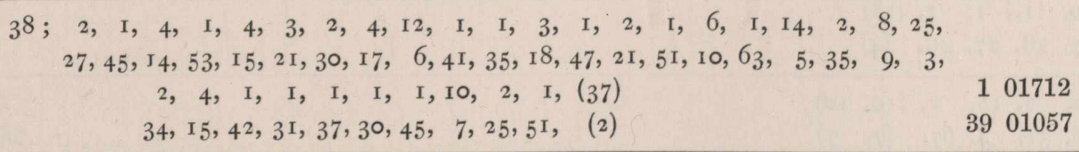 & $\begin{array}{l}8916397276 \\
73734728288\end{array}$ & $\begin{array}{ll}23871 & 56715 \\
54875 & 60183\end{array}$ & 36737 \\
\hline 1472 & $\begin{array}{r}3^{8} ; \quad 2, \quad \mathrm{I}, \quad(2) \\
28, \quad 4 \mathrm{I},(23)\end{array}$ & & & $\begin{array}{r}30 \\
1151\end{array}$ \\
\hline 1473 & $\begin{array}{r}38 ; 2, \quad 1, \quad 1, \quad 1,2, \quad 1,2, \quad 2,9,5, \quad 1, \quad 3, \quad 1,(24) \\
29,37,32,39,23,43,24,31,8,13,53,16,59,\end{array}$ & & $\begin{array}{r}692736 \\
26586992\end{array}$ & $\begin{array}{l}14220 \\
93399\end{array}$ \\
\hline 1474 & 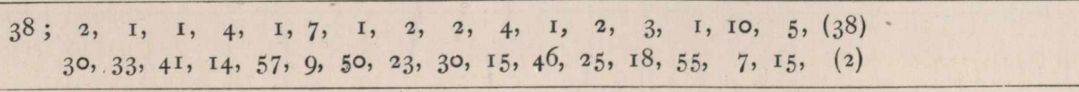 & & $\begin{array}{r}4315108109 \\
9595760507\end{array}$ & $\begin{array}{l}79476 \\
29745\end{array}$ \\
\hline 1475 & $\begin{array}{r}38 ; \quad 2, \quad 6, \quad \mathrm{I}, \quad \mathrm{I}, \quad(2) \\
3^{\mathrm{I}}, 29, \text { II }, 4 \mathrm{I}, 34,(25)\end{array}$ & & & $\begin{array}{l}14628 \\
61799\end{array}$ \\
\hline 1476 & $\begin{array}{r}38 ; \quad 2, \quad 2, \quad 1, \quad 1, \quad 2, \quad 1,3,(8) \\
32, \quad 25,36,37,23,45,20,(9)\end{array}$ & & & $\begin{array}{l}18560 \\
96801\end{array}$ \\
\hline 1477 & 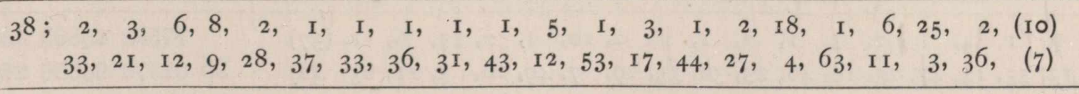 & $\begin{array}{rr}2 & 61633 \\
100 & 55043\end{array}$ & $\begin{array}{ll}72121 & 47062 \\
78017 & 41504\end{array}$ & $\begin{array}{l}22240 \\
36351\end{array}$ \\
\hline 1478 & $\begin{array}{r}38 ; \quad 2, \quad 4,(38) \\
34, \quad 17, \quad(2)\end{array}$ & & & $\begin{array}{r}3114 \\
19717\end{array}$ \\
\hline
\end{tabular}

C. XIII. 
TABLE 1001 TO 1500 - continued.

\begin{tabular}{|c|c|c|c|c|}
\hline 1479 & $\begin{array}{r}38 ; \quad 5, \quad 2, \quad 2, \quad 1, \quad 1, \quad 1, \quad(1) \\
35, \quad 13,30,25,38,33,35,(34)\end{array}$ & & & $\begin{array}{l}14525 \\
4 \\
04376\end{array}$ \\
\hline 1480 & $\begin{array}{r}3^{8} ; 2,8,(\text { 19) } \\
36,9, \quad(4)\end{array}$ & & & $\begin{array}{r}5559 \\
13859\end{array}$ \\
\hline $1481^{*}$ & 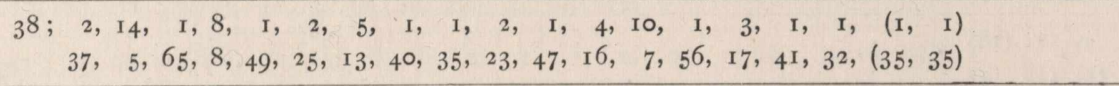 & $\begin{array}{rr}1 & 01945 \\
39 & 23264\end{array}$ & $\begin{array}{l}97513 \\
72655\end{array}$ & $\begin{array}{ll}3 & 61405 \\
5 & 17468\end{array}$ \\
\hline 1482 & $3^{8} ;(2)$ & & & $\begin{array}{r}2 \\
77\end{array}$ \\
\hline 1483 & 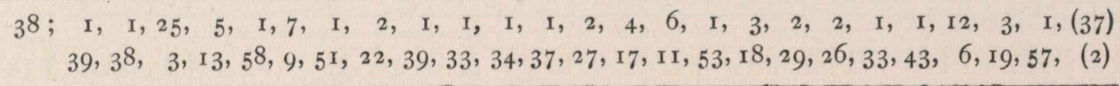 & $\begin{array}{r}412092870570 \\
15869588900942\end{array}$ & $\begin{array}{l}044632 \\
230191\end{array}$ & $\begin{array}{l}238045 \\
181226\end{array}$ \\
\hline 1484 & $\begin{array}{r}3^{8} ; \quad 1, \quad 1,(10) \\
40,37, \quad(7)\end{array}$ & & & $\begin{array}{r}44 \\
1695\end{array}$ \\
\hline 1485 & $\begin{array}{r}3^{8} ; \quad \mathbf{I}, \quad \mathrm{I},(6) \\
4 \mathrm{I}, 3^{6,}(\mathrm{I} \mathrm{I})\end{array}$ & & & $\begin{array}{r}28 \\
1079\end{array}$ \\
\hline 1486 & $\begin{array}{r}38 ; \quad \mathrm{I}, \quad \mathrm{I}, \quad 4, \quad \mathrm{I}, \quad \mathrm{I}, \quad \mathrm{I}, 3, \quad(38) \\
4^{2}, 35, \quad 15,43,30,4 \mathrm{I}, 21,\end{array}$ & & & $\begin{array}{ll}4 & 92228 \\
9 & 74735\end{array}$ \\
\hline 1487 & $\begin{array}{r}38 ; \quad \mathbf{1}, \quad \mathbf{1}, \quad 3, \quad 1, \quad 1, \quad 3, \quad 1, \quad(37) \\
43,34, \quad 19,37,38, \quad 17,59, \quad(2)\end{array}$ & & & $\begin{array}{ll}2 & 05495 \\
9 & 24224\end{array}$ \\
\hline 1488 & $\begin{array}{r}38 ; \quad 1, \quad 1, \quad 2, \quad 1, \quad(5) \\
44,33,23,49,(12)\end{array}$ & & & $\begin{array}{r}315 \\
12151\end{array}$ \\
\hline $1489 *$ & 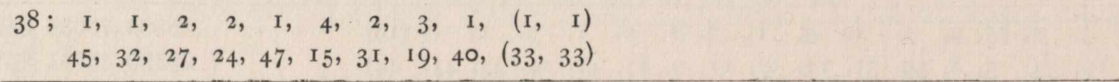 & & & $\begin{array}{l}58145 \\
484332\end{array}$ \\
\hline $1490 *$ & $\begin{array}{r}3^{8} ; \quad \mathrm{I},(\mathrm{I}, \mathrm{I}) \\
4^{6},(3 \mathrm{I}, 3 \mathrm{I})\end{array}$ & & & $\begin{array}{r}5 \\
193\end{array}$ \\
\hline 1491 & $\begin{array}{r}3^{8} ; \quad 1, \quad 1, \quad 1, \quad 1,2, \quad 2, \quad(1) \\
47,30,35,37,26,25,(42)\end{array}$ & 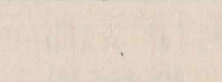 & $y=$ & $\begin{array}{r}1767 \\
68230\end{array}$ \\
\hline 1492 & 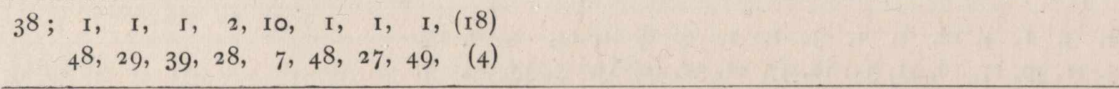 & & & $\begin{array}{l}356270 \\
357849\end{array}$ \\
\hline $1493^{*}$ & 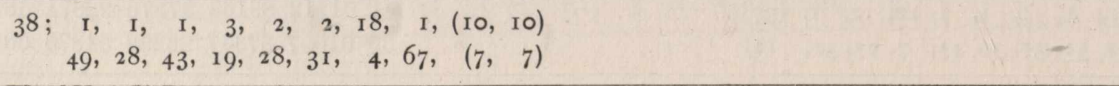 & & $\begin{array}{r}1694 \\
65471\end{array}$ & $\begin{array}{l}41225 \\
100182\end{array}$ \\
\hline 1494 & $\begin{array}{r}3^{8} ; \quad \text { I, I, } 7, \quad \text { I } 5, \quad 5,(38) \\
50,27,47, \text { 10, } 5,25, \quad(2)\end{array}$ & & $\begin{array}{r}440 \\
17009\end{array}$ & $\begin{array}{ll}0 & 05214 \\
9 & 02565\end{array}$ \\
\hline 1495 & $\begin{array}{r}38 ; \quad \mathrm{I},(\mathrm{I}) \\
5 \mathrm{I},(26)\end{array}$ & & & $\begin{array}{r}3 \\
116\end{array}$ \\
\hline 1496 & $\begin{array}{r}3^{8} ; \quad 1, \quad 2,(9) \\
52,25,(8)\end{array}$ & & & $\begin{array}{r}87 \\
3365\end{array}$ \\
\hline 1497 & 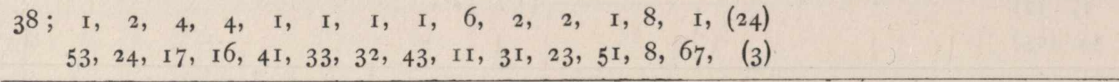 & $\begin{array}{r}58 \\
2251\end{array}$ & $\begin{array}{l}19614 \\
67187\end{array}$ & $\begin{array}{l}430932 \\
751127\end{array}$ \\
\hline 1498 & 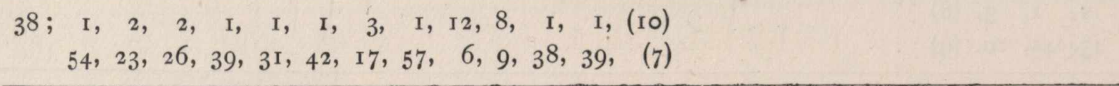 & & $\begin{array}{l}83118 \\
17006\end{array}$ & 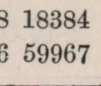 \\
\hline 1499 & $\begin{array}{r}38 ; 1,2, \quad \mathrm{I}, \mathrm{I}, 7,5, \quad \mathrm{I}, 4, \mathrm{I}, 2,3, \mathrm{I}, 2, \mathrm{I}, \mathrm{I}, 2,10, \mathrm{I}, 2,15,6, \mathrm{I},(37) \\
55,22,35,4 \mathrm{I}, 10, \mathrm{I} 3,55, \mathrm{I} 4,47,25,19,46,23,38,35,29,7,49,26,5,1 \mathrm{I}, 65,(2)\end{array}$ & $\begin{array}{rrr}77552 & 05839 \\
30 & 02576 & 94656\end{array}$ & $\begin{array}{l}43067 \\
26953\end{array}$ & $\begin{array}{l}780299 \\
360610\end{array}$ \\
\hline 1500 & $\begin{array}{l}38 ; \quad 1, \quad 2, \quad 1,2, \quad 2, \quad 1,6,2, \quad 1,(18) \\
56,21,44,25,24,49, \quad 11,25,5 \mathrm{I},(4)\end{array}$ & $-\quad$ & $\begin{array}{r}118 \\
4574\end{array}$ & $\begin{array}{l}11844 \\
40751\end{array}$ \\
\hline
\end{tabular}


In connexion with the subject we have a paper, "A Table of the Square Roots of Prime Numbers of the form $4 m+1$ less than 10000 expanded as Periodic Continued Fractions," by C. A. Roberts, with Introduction and Explanation by Artemas Martin, the Mathematical Magazine, vol. II. (No. 7, for October, 1892), pp. 105-120. This extends, in fact, to numbers up to 10501, but only the denominators of the continued fractions (that is, the first lines of Degen's and the present table) are given: thus the entry for 1009 is $31 ; 1,(3,3)$.

The paper just referred to notices errors in Degen's tables for the numbers 853 and 929. For 853 the first line should be

$$
29,4,1,5,1,2,4,1,1,15,19,(2,2) \text {, }
$$

(15 instead of Degen's 14). For 929 the first and second lines should be

$$
\begin{array}{r}
30,2,11,1,2,3,2,7,5, \quad(2,2) \\
1,29,5,40,19,16,25,8,11,(23,23) .
\end{array}
$$

The values of $x, y$ in Table I. and those in Table II. (for the solution of $\left.y^{2}=a x^{2}-1\right)$ are correct for each of the numbers 853 and 929 . 